\title{
Pharmacological interventions for melanoma: Comparative analysis using bayesian meta-analysis
}

\author{
Yang Yang ${ }^{1,2, *}$, Jiaomiao Pei ${ }^{1,}{ }^{*}$, Guozhen $\mathrm{Gao}^{3}$, Zheng Yang ${ }^{4}$, Shuzhong Guo ${ }^{1}$, Bo \\ Yue $^{5}$, Jianhua Qiu ${ }^{5}$ \\ ${ }^{1}$ Department of Plastic and Reconstructive Surgery, Xijing Hospital, Fourth Military Medical University, Xi'an, 710032, China \\ ${ }^{2}$ Medical Insurance and New Rural Cooperative Medical Insurance Administration Center, The 252nd Hospital of PLA, Baoding, \\ 071000, China \\ ${ }^{3}$ Department of Burn and Plastic Surgery, The 253rd Hospital of PLA, Hohhot, 010051, China \\ ${ }^{4}$ The First Brigade of Fourth Military Medical University, Xi'an, 710032, China \\ ${ }^{5}$ Department of Otolaryngology-Head and Neck Surgery, Xijing Hospital, Fourth Military Medical University, Xi'an, 710032, \\ China \\ *These authors have contributed equally to this work \\ Correspondence to: Bo Yue, email: yuebo_mus@163.com \\ Jianhua Qiv, email: jianhuaqivu@yeah.net \\ Keywords: melanoma, chemotherapy, ipilimumab, tremelimumab, nivolumab \\ Received: June 20,2016 Accepted: September 21,2016 Published: October 13, 2016
}

\section{ABSTRACT}

We conducted a network meta-analysis in order to compare different strategies for managing melanoma patients. Electronic databases were searched for eligible randomized trials that compared different strategies in efficacy and tolerability. Five interventions were associated with a significant improvement in PFS over chemotherapy (all HR < 1): Ipilimumab, Tremelimumab, Nivolumab, Pembrolizumab $2 \mathrm{mg} / \mathrm{kg}$ and Ipilimumab + Nivolumab. Three interventions exhibited significantly improved OS results over chemotherapy (all HR $<1$ ): Ipilimumab, Nivolumab and Ipilimumab + Chemotherapy. Four interventions were superior to chemotherapy in CR and PR (all OR > 1): Nivolumab, Pembrolizumab $10 \mathrm{mg} / \mathrm{kg}$, Pembrolizumab $2 \mathrm{mg} / \mathrm{kg}$ and Ipilimumab + Nivolumab. However, the other seven interventions were associated with an increased risk of pruritus compared to chemotherapy (all OR $>1$ ). Ipilimumab, Tremelimumab, Ipilimumab + Nivolumab and Ipilimumab + Chemotherapy might result in a higher risk of diarrhea compared to chemotherapy (all OR $>1$ ). Immune checkpoint therapy or combined interventions might be more effective than chemotherapy for managing melanoma patients. However, chemotherapy appears to be more tolerable than these combined strategies with respect to adverse events.

\section{INTRODUCTION}

Melanoma is a tumor due to melanocytes that develop in various areas such as skin, mucosal membranes, eyes and meninges. It was estimated that 100,000 new melanoma cases were diagnosed in 2012 and the expected number of deaths due to the progression of skin cancer was about 22,000 in 2012 [1]. Despite of the fact that a wide range of therapies have been developed, the prognosis of melanoma is not optimistic since patients tend to have poor responses to traditional treatments such as chemotherapy and radiation therapy [2]. In order to achieve improvement, new approaches have been advocated.
Chemotherapy is usually involved in the systemic treatment of melanoma but it has limited effectiveness [3]. On the other hand, a number of immunotherapy and targeted therapy agents have been authorized to improve the survival status of melanoma patients [4]. Antibodies have been introduced into clinical practices to stimulate the immune system by enhancing anti-tumor responses [5]. Ipilimumab is an antibody able to block the co-inhibitory receptor cytotoxic T-lymphocyte antigen-4 (CTLA-4) and the inhibition of CTLA-4 contributes to a global activation of the immune system and thereby improving the survival status of melanoma patients [5-7]. Emerging data have suggested that synergetic effects can be generated by combining two 
different treatment strategies simultaneously [8]. For instance, an abscopal effect has been demonstrated by introducing both radiation and Ipilimumab [9]. Besides that, combining chemotherapy with checkpoint inhibitors such as Ipilimumab represents a novel way to optimize the corresponding effect of checkpoint inhibitors on melanoma patients. The success of Ipilimumab has triggered the development of other immune-modulating antibodies.

The use of Tremelimumab as an immune checkpoint therapy is still in progress, but it has exhibited some effectiveness in metastatic melanoma and other cancers [10]. As suggested by Canniff et al., the effect of Tremelimumab does not depend on the disease stage or cancer type and Tremelimumab is able to enhance the production of IL-2 in T-cells among both healthy controls and cancer patients with solid tumors [10]. However, it appears that Tremelimumab does not exhibit compelling results in phase III trials and hence potential factors that have significant influence on Tremelimumab plasma exposure should be further investigated [11]. Nivolumab is another immune checkpoint inhibitor approved for managing metastatic melanoma, squamous cell lung cancer, and renal cell cancer [12]. Although Nivolumab is generally well-tolerated, some melanoma patients experienced severe pneumonitis after receiving Nivolumab treatment [12]. Moreover, combining different immune checkpoint inhibitors such as Ipilimumab and Nivolumab has been approved by FDA recently and the combined strategy appeared to outperform each monotherapy with respect to response rate and PFS [13]. However, the major drawbacks of using these checkpoint inhibitors simultaneously are the increased level of toxicity and more adverse events such as rash, pneumonitis, diarrhea and colitis [14].

Apart from CTLA-4, programmed death 1 (PD1) is another well-known immune checkpoint protein which exhibits distinctive mechanisms in cells [15]. Unlike CTLA-4, the PD-1 pathways are able to regulate immune responses in tissues selectively [16] and hence several antibodies targeting PD-1 have been developed in various clinical stages. Pembrolizumab is a humanized monoclonal antibody that inhibits the interaction between PD-1 on T cells, thereby triggering antitumor immune responses related to the PD-1 pathways [15]. As suggested by a randomized cohort study in which patients experienced Ipilimumab-refractory melanoma, the objective response rate $(26 \%)$ is equivalent for those who received Pembrolizumab at the dosage of $2 \mathrm{mg} /$ $\mathrm{kg}$ or $10 \mathrm{mg} / \mathrm{kg}$ every three weeks [17]. One strength of Pembrolizumab is that it is well-tolerated without clear evidence of increased toxicity due to the increase in dosage [18]. However, comparing the efficacy and tolerability between Pembrolizumab and Nivolumab is challenging since they were assessed in different patient populations and it is also very hard to determine the optimal treatment duration for Pembrolizumab [15].
Since the number of immune checkpoint inhibitors approved by FDA is increasing, it is critical to differentiate those inhibitors with respect to their efficacy and safety. Our research was inspired by the rapid development and aimed to provide consistent evidence for the selection of interventions. There were two phases in this study. In the first phase, we searched for all the articles about pharmacological interventions introduced to melanoma. In the second phase, we incorporated the approach of network meta-analysis (NMA) to assess the relative efficacy and safety of the following selected interventions: chemotherapy, Ipilimumab, Tremelimumab, Nivolumab, Pembrolizumab (10 $\mathrm{mg} / \mathrm{kg}$ or $2 \mathrm{mg} / \mathrm{kg})$, Ipilimumab + Nivolumab and Ipilimumab + Chemotherapy.

\section{RESULTS}

\section{Literature search and study characteristics}

After ineligible and duplicated studies were removed, 19 studies were incorporated into the analysis with a total sample size of 6,405 subjects [17, 19-36]. All of these included studies were carried out between 2005 and 2015. Information of studies and clinical features of subjects were listed in Table 1. The Jadad scale results of methodological quality were presented in Supplementary Table S1. The comparison networks of interventions for each endpoint were illustrated in Figure 1.

\section{Comparison of effectiveness among melanoma interventions}

Five interventions appeared to be more effective than chemotherapy with respect to PFS. For instance, patients treated with Ipilimumab were associated with an average $34 \%$ reduction in the HR of PFS compared with those treated with chemotherapy $(\mathrm{HR}=0.66,95 \% \mathrm{CrI}=$ 0.44-0.98). The same trend were found in Tremelimumab $(\mathrm{HR}=0.45,95 \% \mathrm{CrI}=0.24-0.85)$, Nivolumab $(\mathrm{HR}=0.39$, $95 \% \mathrm{CrI}=0.26-0.60)$, Pembrolizumab $2 \mathrm{mg} / \mathrm{kg}(\mathrm{HR}=$ $0.64,95 \% \mathrm{CrI}=0.41-0.99)$ and Ipilimumab + Nivolumab $(\mathrm{HR}=0.33,95 \% \mathrm{CrI}=0.19-0.55)$. Furthermore, Nivolumab and Ipilimumab + Nivolumab were more effective than Ipilimumab $(\mathrm{HR}=0.60,95 \% \mathrm{CrI}=0.39$ $0.90 ; \mathrm{HR}=0.50,95 \% \mathrm{CrI}=0.33-0.76)$ with respect to PFS. Patients treated with Ipilimumab + Nivolumab were associated with a decrease in the HR of PFS compared to those treated with Pembrolizumab $10 \mathrm{mg} / \mathrm{kg}(\mathrm{HR}=0.46$, $95 \% \mathrm{CrI}=0.25-0.82)$ or Pembrolizumab $2 \mathrm{mg} / \mathrm{kg}(\mathrm{HR}=$ $0.51,95 \% \mathrm{CrI}=0.27-0.97)$. Finally, increasing the dose of Pembrolizumab appeared to have no significant effect on the HR of melanoma patients with respect to PFS (HR = $0.89,95 \% \mathrm{CrI}=0.63-1.26$; Table 2, Figure 2).

Three interventions exhibited more compelling OS results in reference to chemotherapy: Ipilimumab $(\mathrm{HR}=0.54$, $95 \% \mathrm{CrI}=0.42-0.70)$, Nivolumab $(\mathrm{HR}=0.42,95 \% \mathrm{CrI}=$ 
Table 1: List of clinical trials testing the use of monoclonal antibodies in melanoma

\begin{tabular}{|c|c|c|c|c|c|c|c|c|c|c|}
\hline \multirow[t]{2}{*}{ Study } & \multirow[t]{2}{*}{ Trial ID } & \multirow[t]{2}{*}{ Phase } & \multirow[t]{2}{*}{ Intervention } & \multirow{2}{*}{$\begin{array}{l}\text { Dosage } \\
(\mathrm{mg} / \mathrm{kg})\end{array}$} & \multirow[t]{2}{*}{$\mathbf{N}$} & \multirow{2}{*}{$\begin{array}{c}\text { Age yrs } \\
\text { (mean, SD) }\end{array}$} & \multirow[t]{2}{*}{ Male, $\%$} & \multicolumn{2}{|c|}{ Metastasis (n, \%) } & \multirow[t]{2}{*}{ Outcomes } \\
\hline & & & & & & & & M0-M1b & M1c & \\
\hline \multirow{2}{*}{$\begin{array}{l}\text { Weber, 2015, } \\
\text { USA }\end{array}$} & NCT01721746 & III & Nibolumab & 3 & 272 & $59(16)$ & $176(65)$ & & $203(75)$ & 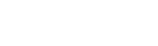 \\
\hline & & & Chemotherapy & & 133 & $62(14)$ & $85(64)$ & & 102(77) & \\
\hline \multirow{3}{*}{$\begin{array}{l}\text { Robert, 2015, } \\
\text { France }\end{array}$} & NCT01866319 & III & Pembrolizumab & 10 & 279 & $61(18)$ & $161(58)$ & $94(35)$ & $179(65)$ & 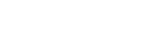 \\
\hline & & & Pembrolizumab & 10 & 277 & $63(17)$ & $174(63)$ & $84(32)$ & $189(68)$ & \\
\hline & & & Ipilimumab & 3 & 278 & $62(18)$ & $162(58)$ & $96(35)$ & $177(65)$ & \\
\hline \multirow{2}{*}{$\begin{array}{l}\text { Robert, 2015, } \\
\text { France }\end{array}$} & NCT01721772 & III & Nivolumab & 3 & 210 & $64(17)$ & $121(58)$ & $82(39)$ & $126(61)$ & 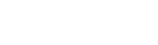 \\
\hline & & & Chemotherapy & & 208 & $66(15)$ & $125(60)$ & $81(39)$ & $127(61)$ & \\
\hline \multirow{3}{*}{$\begin{array}{l}\text { Ribas, 2015, } \\
\text { USA }\end{array}$} & NCT01704287 & II & Pembrolizumab & 2 & 180 & $62(18)$ & $104(58)$ & $32(17)$ & 148(83) & 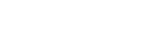 \\
\hline & & & Pembrolizumab & 10 & 181 & $60(16)$ & $109(60)$ & $31(17)$ & $150(83)$ & \\
\hline & & & Chemotherapy & & 179 & $63(15)$ & $114(64)$ & $32(17)$ & $147(83)$ & \\
\hline \multirow{2}{*}{$\begin{array}{l}\text { Postow, 2015, } \\
\text { USA }\end{array}$} & NCT01927419 & III/IV & Ipilimumab+Nivolumab & $3+1$ & 72 & $64(15)$ & $63(66)$ & $50(53)$ & $44(47)$ & 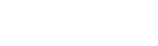 \\
\hline & & & Ipilimumab & 3 & 37 & $67(12)$ & $32(68)$ & $25(55)$ & $21(45)$ & \\
\hline \multirow{2}{*}{$\begin{array}{l}\text { Miao, 2015, } \\
\text { Italy }\end{array}$} & CA184-024 & III & Ipilimumab + Chemotherapy & 10 & 40 & $58(13)$ & $23(62)$ & $22(55)$ & $18(45)$ & 曲 \\
\hline & & & Chemotherapy & & 20 & $61(11)$ & $9(45)$ & $15(75)$ & $5(25)$ & \\
\hline \multirow{3}{*}{$\begin{array}{l}\text { Larkin, 2015, } \\
\text { USA }\end{array}$} & NCT01844505 & III & Nivolumab & 3 & 316 & $59(16)$ & $202(64)$ & $312(42)$ & $184(58)$ & 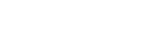 \\
\hline & & & Ipilimumab+Nivolumab & $3+1$ & 314 & $59(18)$ & 206(66) & $133(42)$ & $181(58)$ & \\
\hline & & & Ipilimumab & 3 & 315 & $61(18)$ & $202(64)$ & 132(42) & $183(58)$ & \\
\hline \multirow{2}{*}{$\begin{array}{l}\text { Eggmont, } \\
\text { 2015, France }\end{array}$} & NCT00636168 & III & Ipilimumab & & 475 & $51(16)$ & $296(62)$ & & & QWXXXXV \\
\hline & & & Chemotherapy & & 476 & $52(15)$ & 293(62) & & & \\
\hline \multirow{2}{*}{$\begin{array}{l}\text { Robert, 2014, } \\
\text { France }\end{array}$} & NCT01295827 & I & Pembrolizumab & 2 & 89 & $57(17)$ & $48(54)$ & $31(35)$ & $58(65)$ & 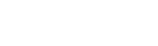 \\
\hline & & & Pembrolizumab & 10 & 84 & $61(15)$ & $57(68)$ & $32(38)$ & $52(62)$ & \\
\hline \multirow{2}{*}{$\begin{array}{l}\text { Hodi, 2014, } \\
\text { USA }\end{array}$} & NCT01134614 & III/IV & Ipilimumab+Chemotherapy & & 123 & $61(15)$ & $85(69)$ & $33(27)$ & $61(50)$ & 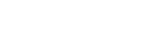 \\
\hline & & & Ipilimumab & & 122 & $64(17)$ & $78(64)$ & $31(25)$ & $60(49)$ & \\
\hline \multirow{2}{*}{$\begin{array}{l}\text { Ribas, 2013, } \\
\text { USA }\end{array}$} & NCT00257205 & III & Tremelimumab & 15 & 328 & $57(17)$ & $190(58)$ & $121(37)$ & $188(57)$ & 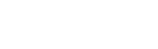 \\
\hline & & & Chemotherapy & & 327 & $56(17)$ & $182(56)$ & $119(36)$ & 194(59) & \\
\hline \multirow{3}{*}{$\begin{array}{l}\text { Millward, } \\
2013, \\
\text { Australia }\end{array}$} & & IV & Tremelimumab & 6 & 3 & & $14(67)$ & $8(38)$ & $9(43)$ & QXXXXV \\
\hline & & & Tremelimumab & 10 & 6 & & & & & \\
\hline & & & Tremelimumab & 15 & 6 & & & & & \\
\hline \multirow{2}{*}{$\begin{array}{l}\text { Robert, 2011, } \\
\text { France }\end{array}$} & NCT00324155 & III/IV & Ipilimumab+Chemotherapy & 10 & 250 & 58 & $152(61)$ & 107(43) & $143(57)$ & 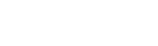 \\
\hline & & & Chemotherapy & & 252 & 56 & $149(59)$ & $113(45)$ & $139(55)$ & \\
\hline
\end{tabular}

(Continued) 


\begin{tabular}{|c|c|c|c|c|c|c|c|c|c|c|}
\hline \multirow[t]{2}{*}{ Study } & \multirow[t]{2}{*}{ Trial ID } & \multirow[t]{2}{*}{ Phase } & \multirow[t]{2}{*}{ Intervention } & \multirow{2}{*}{$\begin{array}{l}\text { Dosage } \\
\text { (mg/kg) }\end{array}$} & \multirow[t]{2}{*}{$\mathbf{N}$} & \multirow{2}{*}{$\begin{array}{c}\text { Age yrs } \\
\text { (mean, SD) }\end{array}$} & \multirow[t]{2}{*}{ Male, $\%$} & \multicolumn{2}{|c|}{ Metastasis (n, \%) } & \multirow[t]{2}{*}{ Outcomes } \\
\hline & & & & & & & & M0-M1b & M1c & \\
\hline \multirow{2}{*}{$\begin{array}{l}\text { Hersh, 2011, } \\
\text { USA }\end{array}$} & NCT00050102 & II & Ipilimumab & 3 & 40 & $66(14)$ & $21(57)$ & $16(43)$ & $21(57)$ & 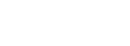 \\
\hline & & & Ipilimumab+Chemotherapy & 3 & 36 & $60(14)$ & $26(74)$ & $18(51)$ & $16(46)$ & \\
\hline \multirow{2}{*}{$\begin{array}{l}\text { Hamid, 2011, } \\
\text { USA }\end{array}$} & NCT00261365 & II & Ipilimumab & 3 & 40 & $54(14)$ & $28(70)$ & $18(45)$ & $22(55)$ & $\mathrm{W}$ \\
\hline & & & Ipilimumab & 10 & 42 & $56(15)$ & $24(57)$ & $14(33)$ & $28(67)$ & \\
\hline \multirow{2}{*}{$\begin{array}{l}\text { Wolchok, } \\
\text { 2011, USA }\end{array}$} & NCT00289640 & III/IV & Ipilimumab & 10 & 73 & $59(15)$ & $52(71)$ & $28(39)$ & $45(62)$ & $\mathrm{W} \times \mathrm{W} \times \mathrm{W} \times \mathrm{x}$ \\
\hline & & & Ipilimumab & 3 & 72 & $59(12)$ & $48(67)$ & $36(50)$ & $36(50)$ & \\
\hline \multirow{2}{*}{$\begin{array}{l}\text { Weber, 2009, } \\
\text { UK }\end{array}$} & & III/IV & Ipilimumab + Chemotherapy & 10 & 58 & $58(13)$ & $43(74)$ & $30(52)$ & $28(48)$ & $\mathrm{AWD}$ \\
\hline & & & Ipilimumab & 10 & 57 & $61(16)$ & $38(67)$ & $28(49)$ & $29(51)$ & \\
\hline \multirow{4}{*}{$\begin{array}{l}\text { Camacho, } \\
\text { 2009, Finland }\end{array}$} & NCT0086489 & I & Tremelimumab & 6 & 3 & & & & & 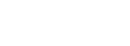 \\
\hline & & & Tremelimumab & 10 & 22 & & & & & \\
\hline & & II & Tremelimumab & 10 & 44 & $61(15)$ & $26(59)$ & $14(32)$ & $29(66)$ & \\
\hline & & & Tremelimumab & 15 & 46 & $54(16)$ & $30(65)$ & $11(25)$ & $32(73)$ & \\
\hline \multirow{2}{*}{$\begin{array}{l}\text { Ribas, 2005, } \\
\text { USA }\end{array}$} & - & I & Ipilimumab & 3 & 9 & $54(12)$ & $27(69)$ & & & $\mathrm{AMD}$ \\
\hline & & & Ipilimumab & 10 & 11 & & & & & \\
\hline
\end{tabular}

Metastasis Category: M0=no distant metastasis; M1a=metastasis to skin, subcutaneous tissues, or distant lymph nodes; M1b=metastasis to lung; M1c=to

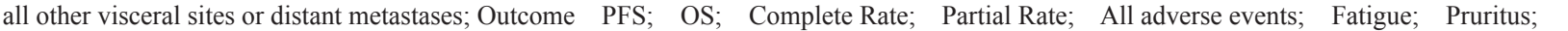
Rash; $₫$ Diarrhea; $₫$ Nausea.

(1) OS

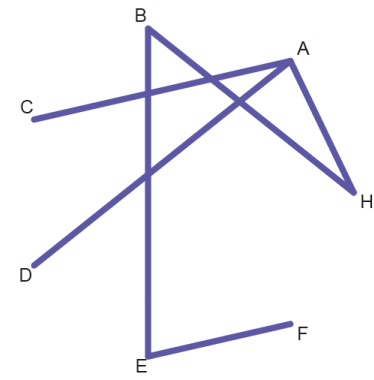

(4) PR

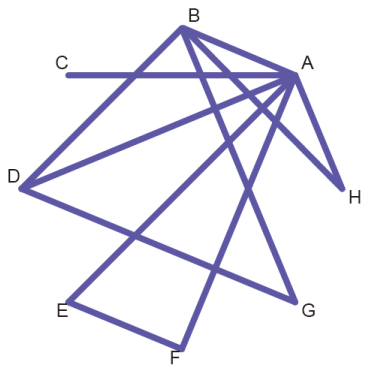

(2) PFS

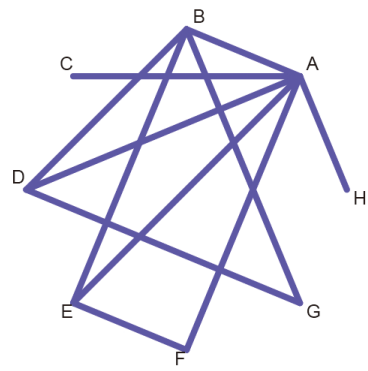

(5) Fatigue and Nausea

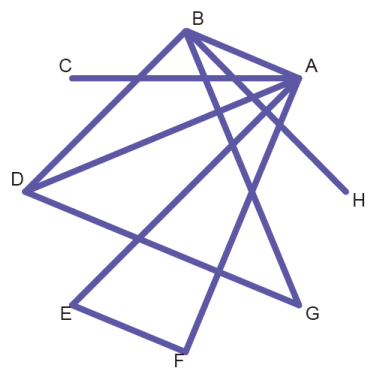

(3) $C R$ and $A A E$

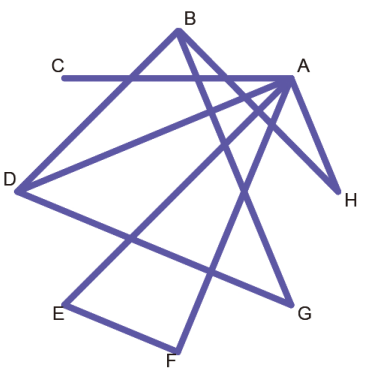

(6) Prurituse, Diarrhea and Rush

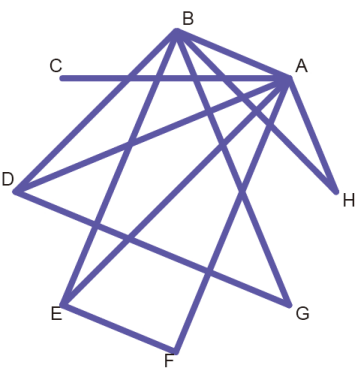

Figure 1: Network diagram. Each node represents a melanoma therapy. 
Table 2: Main NMA outcomes for melanoma

\begin{tabular}{|c|c|c|c|c|c|c|c|c|}
\hline \multirow[t]{8}{*}{ PFS } & $\mathbf{A}$ & $1.52(1.02,2.27)$ & $2.24(1.18,4.23)$ & $2.55(1.66,3.91)$ & $1.39(0.93,2.09)$ & $1.56(1.01,2.44)$ & $3.05(1.8,5.16)$ & $1.32(0.71,2.42)$ \\
\hline & $0.66(0.44,0.98)$ & B & $1.47(0.69,3.13)$ & $1.68(1.11,2.53)$ & $0.92(0.58,1.44)$ & $1.03(0.61,1.73)$ & $2.01(1.32,3.05)$ & $0.87(0.42,1.80)$ \\
\hline & $0.45(0.24,0.85)$ & $0.68(0.32,1.44)$ & $\mathrm{C}$ & $1.14(0.53,2.46)$ & $0.62(0.29,1.33)$ & $0.7(0.32,1.52)$ & $1.36(0.6,3.12)$ & $0.59(0.24,1.42)$ \\
\hline & $0.39(0.26,0.6)$ & $0.60(0.39,0.90)$ & $0.88(0.41,1.89)$ & D & $0.55(0.32,0.93)$ & $0.61(0.35,1.09)$ & $1.2(0.76,1.88)$ & $0.52(0.24,1.09)$ \\
\hline & $0.72(0.48,1.08)$ & $1.09(0.69,1.72)$ & $1.61(0.75,3.42)$ & $1.83(1.07,3.12)$ & $\mathbf{E}$ & $1.12(0.79,1.59)$ & $2.19(1.22,3.93)$ & $0.94(0.45,1.97)$ \\
\hline & $0.64(0.41,0.99)$ & $0.97(0.58,1.63)$ & $1.43(0.66,3.11)$ & $1.63(0.92,2.9)$ & $0.89(0.63,1.26)$ & $\mathbf{F}$ & $1.95(1.03,3.67)$ & $0.84(0.40,1.79)$ \\
\hline & $0.33(0.19,0.55)$ & $0.5(0.33,0.76)$ & $0.73(0.32,1.68)$ & $0.84(0.53,1.31)$ & $0.46(0.25,0.82)$ & $0.51(0.27,0.97)$ & G & $0.43(0.19,0.97)$ \\
\hline & $0.76(0.41,1.4)$ & $1.15(0.56,2.40)$ & $1.7(0.7,4.11)$ & $1.94(0.92,4.09)$ & $1.06(0.51,2.21)$ & $1.19(0.56,2.53)$ & $2.32(1.03,5.19)$ & $\mathbf{H}$ \\
\hline \multirow[t]{8}{*}{ OS } & $\mathbf{A}$ & $1.84(1.43,2.36)$ & $1.13(0.97,1.32)$ & $2.38(1.39,4.07)$ & $1.27(0.87,1.84)$ & $1.38(0.76,2.52)$ & - & $1.39(1.14,1.69)$ \\
\hline & $0.54(0.42,0.7)$ & B & $0.62(0.46,0.83)$ & $1.3(0.72,2.34)$ & $0.69(0.52,0.91)$ & $0.75(0.44,1.30)$ & - & $0.76(0.65,0.89)$ \\
\hline & $0.88(0.76,1.04)$ & $1.62(1.21,2.18)$ & $\mathrm{C}$ & $2.11(1.21,3.68)$ & $1.12(0.75,1.68)$ & $1.22(0.66,2.27)$ & - & $1.23(0.96,1.58)$ \\
\hline & $0.42(0.25,0.72)$ & $0.77(0.43,1.39)$ & $0.47(0.27,0.83)$ & D & $0.53(0.28,1.02)$ & $0.58(0.26,1.30)$ & - & $0.58(0.33,1.03)$ \\
\hline & $0.79(0.54,1.14)$ & $1.45(1.1,1.91)$ & $0.89(0.6,1.34)$ & $1.88(0.98,3.61)$ & $\mathbf{E}$ & $1.09(0.68,1.75)$ & - & $1.1(0.80,1.51)$ \\
\hline & $0.72(0.4,1.32)$ & $1.33(0.77,2.3)$ & $0.82(0.44,1.52)$ & $1.72(0.77,3.86)$ & $0.92(0.57,1.47)$ & $\mathbf{F}$ & - & $1.01(0.57,1.78)$ \\
\hline & - & - & - & - & - & - & G & - \\
\hline & $0.72(0.59,0.87)$ & $1.32(1.13,1.55)$ & $0.81(0.63,1.04)$ & $1.71(0.97,3.03)$ & $0.91(0.66,1.25)$ & $0.99(0.56,1.76)$ & - & $\mathbf{H}$ \\
\hline \multirow[t]{8}{*}{ CR } & $\mathbf{A}$ & $1.48(0.31,7.94)$ & $1.32(0.30,6.45)$ & $8.09(2.28,30.0)$ & $7.36(1.54,37.9)$ & $6.50(1.31,32.9)$ & $10.8(2.29,68.3)$ & $3.48(0.82,15.6)$ \\
\hline & $0.68(0.13,3.27)$ & B & $0.89(0.10,8.66)$ & $5.58(1.61,20.0)$ & $4.98(0.53,50.1)$ & $4.20(0.43,42.4)$ & $7.43(2.51,27.3)$ & $2.21(0.46,12.0)$ \\
\hline & $0.76(0.16,3.35)$ & $1.12(0.12,10.03)$ & C & $6.08(0.76,45.1)$ & $5.77(0.61,49.1)$ & $5.03(0.51,40.8)$ & $8.28(0.85,86.2)$ & $2.59(0.31,22.0)$ \\
\hline & $0.12(0.03,0.44)$ & $0.18(0.05,0.62)$ & $0.16(0.02,1.32)$ & D & $0.94(0.11,7.13)$ & $0.77(0.10,6.63)$ & $1.34(0.41,5.14)$ & $0.42(0.08,2.05)$ \\
\hline & $0.14(0.03,0.65)$ & $0.20(0.02,1.89)$ & $0.17(0.02,1.64)$ & $1.06(0.14,9.07)$ & $\mathbf{E}$ & $0.84(0.25,2.83)$ & $1.46(0.16,16.5)$ & $0.45(0.05,4.03)$ \\
\hline & $0.15(0.03,0.76)$ & $0.24(0.02,2.31)$ & $0.20(0.02,1.98)$ & $1.30(0.15,10.4)$ & $1.18(0.35,4.01)$ & $\mathbf{F}$ & $1.77(0.17,19.2)$ & $0.54(0.06,5.13)$ \\
\hline & $0.09(0.01,0.44)$ & $0.13(0.04,0.40)$ & $0.12(0.01,1.18)$ & $0.75(0.19,2.44)$ & $0.68(0.06,6.24)$ & $0.57(0.05,5.80)$ & G & $0.31(0.05,1.82)$ \\
\hline & $0.29(0.06,1.22)$ & $0.45(0.08,2.16)$ & $0.39(0.05,3.20)$ & $2.38(0.49,11.9)$ & $2.21(0.25,19.9)$ & $1.87(0.20,16.9)$ & $3.25(0.55,20.6)$ & $\mathbf{H}$ \\
\hline \multirow[t]{8}{*}{ PR } & $\mathbf{A}$ & $1.44(0.78,2.70)$ & $1.03(0.49,2.23)$ & $3.52(2.37,5.83)$ & $6.47(2.85,15.5)$ & $5.49(2.39,13.9)$ & $6.14(3.43,12.3)$ & $1.53(0.93,2.56)$ \\
\hline & $0.70(0.37,1.29)$ & B & $0.72(0.27,1.83)$ & $2.47(1.44,4.31)$ & $4.46(1.56,13.7)$ & $3.86(1.34,12.6)$ & $4.29(2.59,7.08)$ & $1.08(0.62,1.76)$ \\
\hline & $0.97(0.45,2.06)$ & $1.40(0.55,3.71)$ & $\mathrm{C}$ & $3.49(1.44,8.70)$ & $6.34(2.00,21.3)$ & $5.36(1.69,18.5)$ & $6.01(2.28,17.0)$ & $1.47(0.61,3.89)$ \\
\hline & $0.28(0.17,0.42)$ & $0.40(0.23,0.70)$ & $0.29(0.11,0.70)$ & D & $1.89(0.69,4.82)$ & $1.59(0.57,4.20)$ & $1.73(1.04,2.98)$ & $0.44(0.24,0.77)$ \\
\hline & $0.15(0.06,0.35)$ & $0.22(0.07,0.64)$ & $0.16(0.05,0.50)$ & $0.53(0.21,1.46)$ & $\mathbf{E}$ & $0.88(0.50,1.48)$ & $0.96(0.32,2.88)$ & $0.24(0.08,0.64)$ \\
\hline & $0.18(0.07,0.42)$ & $0.26(0.08,0.74)$ & $0.19(0.05,0.59)$ & $0.63(0.24,1.75)$ & $1.14(0.68,1.99)$ & $\mathbf{F}$ & $1.14(0.36,3.40)$ & $0.28(0.09,0.76)$ \\
\hline & $0.16(0.08,0.29)$ & $0.23(0.14,0.39)$ & $0.17(0.06,0.44)$ & $0.58(0.34,0.96)$ & $1.04(0.35,3.13)$ & $0.87(0.29,2.79)$ & G & $0.25(0.13,0.47)$ \\
\hline & $0.65(0.39,1.08)$ & $0.93(0.57,1.62)$ & $0.68(0.26,1.65)$ & $2.30(1.31,4.16)$ & $4.25(1.56,12.9)$ & $3.60(1.32,11.6)$ & $4.03(2.15,7.96)$ & $\mathbf{H}$ \\
\hline \multirow[t]{8}{*}{$\mathbf{A A E}$} & $\mathbf{A}$ & $2.32(0.58,7.84)$ & $2.37(0.38,14.33)$ & $1.31(0.44,4.10)$ & $0.80(0.13,4.97)$ & $0.60(0.10,3.42)$ & $5.36(0.79,39.98)$ & $3.95(0.79,18.57)$ \\
\hline & $0.43(0.13,1.72)$ & B & $1.00(0.12,10.92)$ & $0.56(0.16,2.63)$ & $0.34(0.04,3.42)$ & $0.25(0.03,2.63)$ & $2.34(0.44,14.64)$ & $1.72(0.37,8.81)$ \\
\hline & $0.42(0.07,2.64)$ & $1.00(0.09,8.12)$ & $\mathrm{C}$ & $0.56(0.06,4.87)$ & $0.34(0.02,4.22)$ & $0.26(0.02,3.20)$ & $2.30(0.15,32.86)$ & $1.68(0.14,18.08)$ \\
\hline & $0.76(0.24,2.30)$ & $1.77(0.38,6.44)$ & $1.80(0.21,16.06)$ & D & $0.61(0.07,4.87)$ & $0.46(0.05,3.75)$ & $4.05(0.63,26.92)$ & $3.01(0.48,17.77)$ \\
\hline & $1.24(0.20,7.90)$ & $2.94(0.29,23.53)$ & $2.94(0.24,41.05)$ & $1.63(0.21,14.54)$ & $\mathbf{E}$ & $0.74(0.13,4.50)$ & $6.65(0.50,94.12)$ & $6.70(0.57,68.03)$ \\
\hline & $1.68(0.29,9.53)$ & $3.94(0.38,31.62)$ & $3.92(0.31,48.92)$ & $2.19(0.27,18.46)$ & $1.36(0.22,7.59)$ & $\mathbf{F}$ & $9.02(0.67,23.75)$ & $0.73(0.07,6.92)$ \\
\hline & $0.19(0.03,1.27)$ & $0.43(0.07,2.28)$ & $0.43(0.03,6.61)$ & $0.25(0.04,1.59)$ & $0.15(0.01,1.99)$ & $0.11(0.01,1.48)$ & G & - \\
\hline & $0.25(0.05,1.26)$ & $0.58(0.11,2.72)$ & $0.60(0.06,7.09)$ & $0.33(0.06,2.09)$ & $0.20(0.02,2.25)$ & $0.15(0.01,1.74)$ & $1.37(0.14,13.36)$ & $\mathbf{H}$ \\
\hline
\end{tabular}

Intervention: A: Chemotherapy; B: Ipilimumab; C: Tremelimumab; D: Nivolumab; E: Pembrolizumab 10 mg/kg; F: Pembrolizumab 2 mg/kg; G: Ipilimumab+Nivolumab; H: Ipilimumab+Chemotherapy; Outcomes: PFS: Progression Free Survival; OS: Overall Survival; CR: Complete Rate; PR: Partial Rate; AAE: All Adverse Events. 
0.25-0.72) and Ipilimumab + Chemotherapy $(\mathrm{HR}=0.72,95 \%$ $\mathrm{CrI}=0.59-0.87)$. Besides chemotherapy, three interventions were less effective than Ipilimumab in OS: Tremelimumab $(\mathrm{HR}=1.62,95 \% \mathrm{CrI}=1.21-2.18)$, Pembrolizumab $10 \mathrm{mg} /$ $\mathrm{kg}(\mathrm{HR}=1.45,95 \% \mathrm{CrI}=1.1-1.91)$ and Ipilimumab + Chemotherapy $(\mathrm{HR}=1.32,95 \% \mathrm{CrI}=1.13-1.55)$. Results from NMA also provided evidence that melanoma patients treated with Nivolumab were associated with a decrease in the HR in relation to those treated with Tremelimumab (HR $=0.47,95 \% \mathrm{CrI}=0.27-0.83$; Table 2, Figure 2).

Compared to chemotherapy, four interventions appeared to have stronger efficacy: Nivolumab (OR = 8.09, 95\% CrI = 2.28-30.0), Pembrolizumab $10 \mathrm{mg} /$ $\mathrm{kg}(\mathrm{OR}=7.36,95 \% \mathrm{CrI}=1.54-37.9)$, Pembrolizumab $2 \mathrm{mg} / \mathrm{kg}(\mathrm{OR}=6.5,95 \% \mathrm{CrI}=1.31-32.9)$, Ipilimumab + Nivolumab $(\mathrm{OR}=10.8,95 \% \mathrm{CrI}=2.29-68.3)$. Two interventions were significantly better than Ipilimumab with respect to $\mathrm{CR}$ : Nivolumab $(\mathrm{OR}=5.58,95 \% \mathrm{CrI}=$ 1.61-20.0) and Ipilimumab + Nivolumab (OR $=7.43$, $95 \% \mathrm{CrI}=2.51-27.3)$. Likewise, a few significant results were obtained from NMA with respect to the endpoint of PR. Chemotherapy were less effective than the following four interventions: Nivolumab $(\mathrm{OR}=0.28,95 \% \mathrm{CrI}=$ $0.17-0.42)$, Pembrolizumab $10 \mathrm{mg} / \mathrm{kg}(\mathrm{OR}=0.15,95 \%$ $\mathrm{CrI}=0.06-0.35)$, Pembrolizumab $2 \mathrm{mg} / \mathrm{kg}(\mathrm{OR}=0.18$, $95 \% \mathrm{CrI}=0.07-0.42$ ) and Ipilimumab + Nivolumab $(\mathrm{OR}=0.16,95 \% \mathrm{CrI}=0.08-0.29)$. Besides, the above four interventions also exhibited stronger effectiveness than Ipilimumab as well as Tremelimumab (all OR $<1$ ). Patients treated with Nivolumab, Pembrolizumab $10 \mathrm{mg} /$ $\mathrm{kg}$, Pembrolizumab $2 \mathrm{mg} / \mathrm{kg}$ and Ipilimumab + Nivolumab appeared to have more optimistic PR results compared to those treated with Ipilimumab + Chemotherapy (all OR $>1$; Table 2, Figure 3).
(1) OS

\section{Treatment}

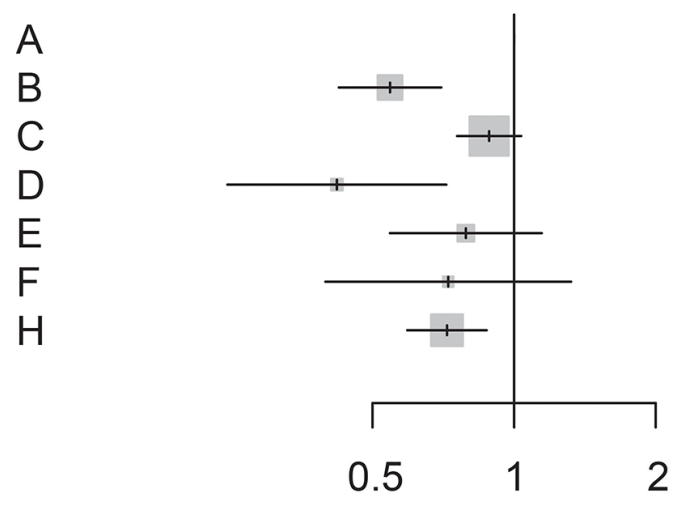

\section{(2) PFS}

\section{Treatment}

A
$B$
C
$D$
E
$F$
$G$
$H$
HR (95\%Crl)

1.00

$0.54(0.42 ; 0.70)$

$0.88(0.76 ; 1.04)$

$0.42(0.25 ; 0.72)$

$0.79(0.54 ; 1.14)$

$0.72(0.40 ; 1.32)$

$0.72(0.59 ; 0.87)$

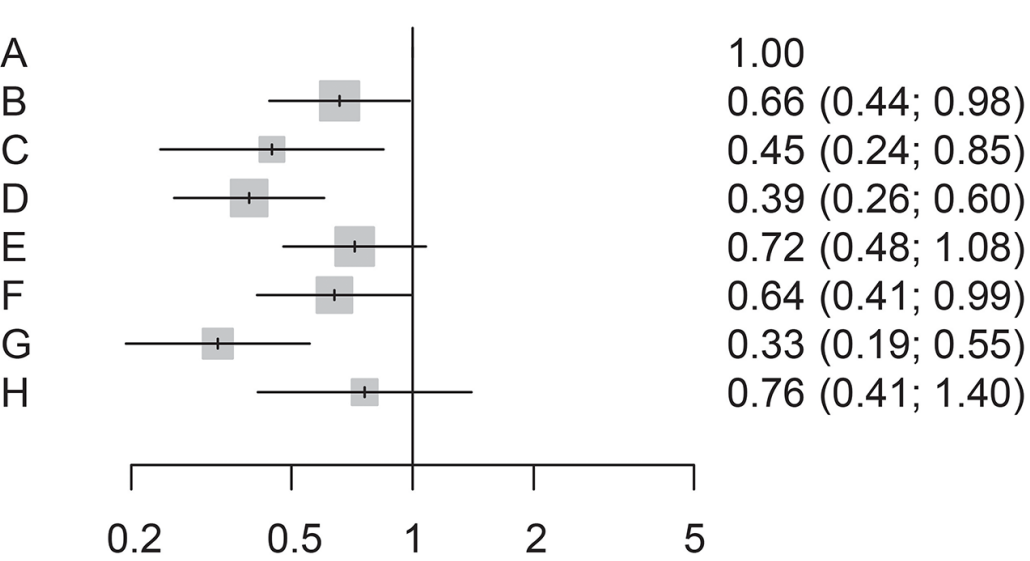

Figure 2: Forest plots for mixed treatment comparison of prognostic data overall survival \& progression-free survival. 


\section{Comparison of adverse events among interventions}

We also compared the tolerability of interventions assessed by different adverse events. Firstly, the risk of fatigue did not appear to be significantly different among

(1) CR

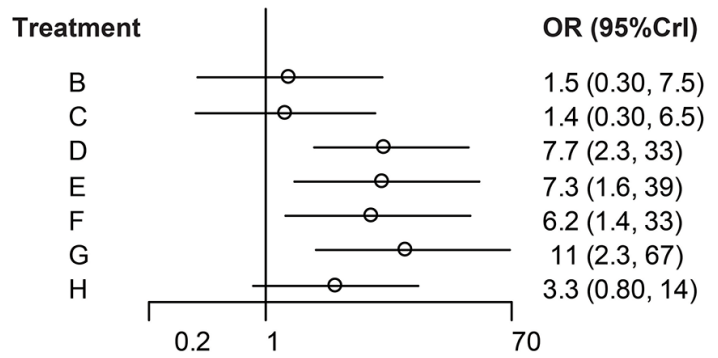

(3) AAE

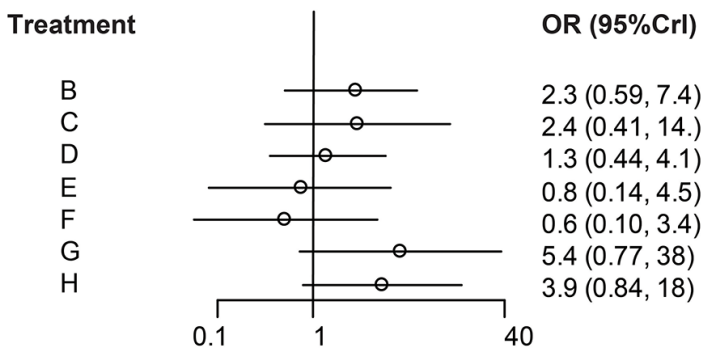

(5) Prurituse

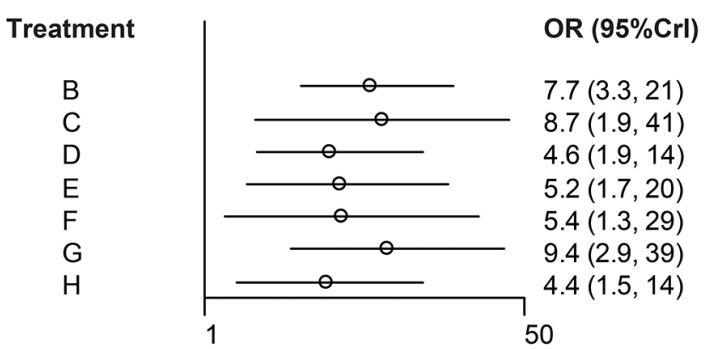

\section{(7) Nausea}

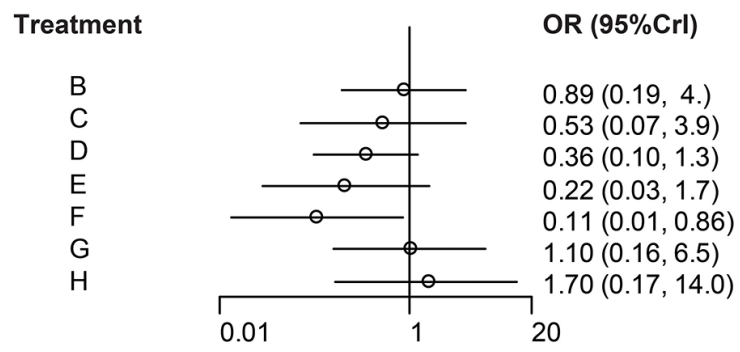

melanoma patients treated with these interventions (all 95\% CrI includes 1). However, patients treated with the other seven interventions were associated with an increased risk of Pruritus compared to those treated with chemotherapy: Ipilimumab $(\mathrm{OR}=7.62,95 \% \mathrm{CrI}$ $=3.33-20.42)$, Tremelimumab $(\mathrm{OR}=8.84,95 \% \mathrm{CrI}=$

\section{(2) PR}

Treatment

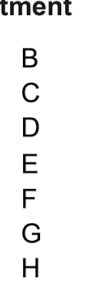

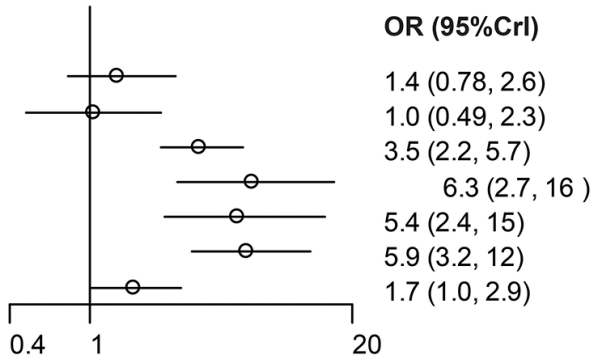

\section{(4) Fatigue}

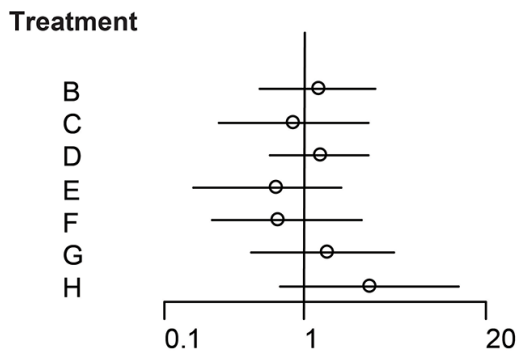

OR (95\%Crl)

$1.3(0.48,3.2)$

$0.85(0.25,2.9)$

$1.3(0.57,2.9)$

$0.63(0.16,1.9)$

$0.65(0.22,2.6)$

$1.5(0.42,4.4)$

$3.0(0.67,13)$

\section{(6) Diarrhea}
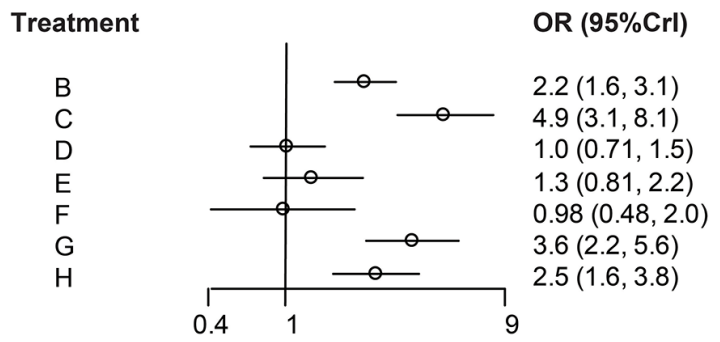

\section{(8) Rush}

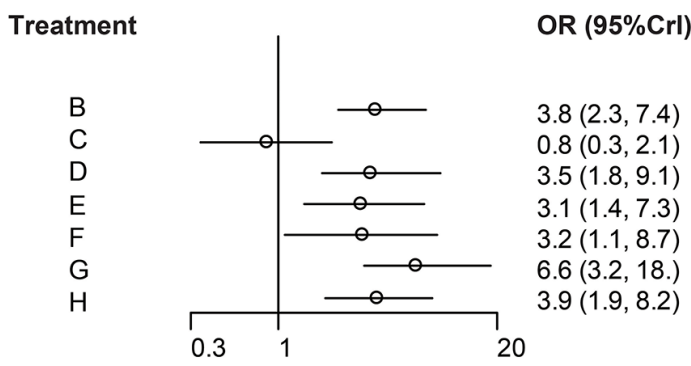

Figure 3: Forest plots for mixed treatment comparison of different outcomes. (1) CR: complete; rate; (2) PR: partial rate; (3) AAE: all adverse events; (4) Fatigue; (5) Pruritus; (6) Diarrhea; (7) Nausea; (8) Rush. 
1.96-42.1), Nivolumab $(\mathrm{OR}=4.62,95 \% \mathrm{CrI}=1.98-$ 13.7), Pembrolizumab $10 \mathrm{mg} / \mathrm{kg}(\mathrm{OR}=5.21,95 \% \mathrm{CrI}=$ 1.78-19.51), Pembrolizumab $2 \mathrm{mg} / \mathrm{kg}(\mathrm{OR}=5.40,95 \%$ $\mathrm{CrI}=1.35-25.58)$, Ipilimumab + Nivolumab $(\mathrm{OR}=9.31$, $95 \% \mathrm{CrI}=3.00-36.8)$ and Ipilimumab + Chemotherapy $(\mathrm{OR}=4.45,95 \% \mathrm{CrI}=1.53-13.4)$ (Table 3, Figure 3$)$. Results from NMA also suggested that the selection of intervention might significantly affect the risk of diarrhea. Ipilimumab, Tremelimumab, Ipilimumab + Nivolumab and Ipilimumab + Chemotherapy might result in a higher risk of diarrhea compared to chemotherapy (all OR > 1). Tremelimumab might result in a higher risk of diarrhea compared to Nivolumab, Pembrolizumab $10 \mathrm{mg} / \mathrm{kg}$, Pembrolizumab $2 \mathrm{mg} / \mathrm{kg}$ and Ipilimumab + Chemotherapy $(\mathrm{OR}>1)$. Melanoma patients treated with Ipilimumab + Nivolumab or Ipilimumab + Chemotherapy were associated with an elevated risk of diarrhea compared to those treated with Nivolumab, Pembrolizumab $10 \mathrm{mg} / \mathrm{kg}$ and Pembrolizumab $2 \mathrm{mg} / \mathrm{kg}$ (all OR > 1; Table 3, Figure 3 ). Another safety measurement of interventions was the risk of rush. Six interventions appeared to result in an increased risk of rush: Ipilimumab $(\mathrm{OR}=3.79$, 95\% CrI $=2.26-7.22)$, Nivolumab $(\mathrm{OR}=3.40,95 \% \mathrm{CrI}$ $=1.83-8.80)$, Pembrolizumab $10 \mathrm{mg} / \mathrm{kg}(\mathrm{OR}=3.03$, $95 \% \mathrm{CrI}=1.43-7.22)$, Pembrolizumab $2 \mathrm{mg} / \mathrm{kg}(\mathrm{OR}=$ $3.06,95 \% \mathrm{CrI}=1.10-8.36)$, Ipilimumab + Nivolumab $(\mathrm{OR}=6.45,95 \% \mathrm{CrI}=2.20-18.20)$ and Ipilimumab + Chemotherapy $(\mathrm{OR}=3.78,95 \% \mathrm{CrI}=1.85-8.05)$. Four interventions including Nivolumab, Pembrolizumab $10 \mathrm{mg} / \mathrm{kg}$, Ipilimumab + Nivolumab and Ipilimumab + Chemotherapy were associated with an increased risk of rush compared to Tremelimumab (all OR $>1$ ).

\section{Assessing consistency between direct and indirect evidence}

Since the consistency model was used in the implementation of the NMA, it was essential to assess the consistency between direct and indirect evidence for each comparison [37]. The output plot conveyed the information about the appropriateness of the consistency model which is confirmed by their corresponding $P$-values. For instance, there was no significant discrepancy between direct evidence and indirect evidence for each comparison with respect to CR $(P$-value $>0.05$, Figure 4$)$. Also, the consistency model is appropriate for comparing interventions with respect to other endpoints including PR (Figure 5), AAE (Figure 6), fatigue (Supplementary Figure S1), pruritus (Supplementary Figure S2), diarrhea (Supplementary Figure S3), nausea (Supplementary Figure S4) and rush (Supplementary Figure S5) (all $P$-value $>0.05$ ). Therefore, we concluded that the consistency model was valid for comparing interventions with respect to the above endpoints.

\section{Ranking of interventions}

One advantage of carrying out a NMA with the Bayesian framework is its ability to produce ranking probabilities which can be used to discriminate interventions with respect to each endpoint. As suggested by the accumulative ranking probability plots and SUCRA values (Table 4, Figure 7), Ipilimumab + Nivolumab exhibited the highest SUCRA with respect to PFS (0.929) and CR (0.857). The standard chemotherapy appeared to have relative weak effectiveness as indicated by lower SUCRA values for PFS, OS, CR and PR whereas such a disadvantage was compensated by its higher SUCRA values with respect to AAE, fatigue, pruritus, diarrhea and rush. On the other hand, Pembrolizumab $10 \mathrm{mg} / \mathrm{kg}$ or $2 \mathrm{mg} /$ $\mathrm{kg}$ appeared to balance between effectiveness and safety since its enhanced efficacy due to increase in dose was not compensated by its increased toxicity. The combined therapy of Ipilimumab + Nivolumab appeared to have higher SUCRA values with respect to PFS, CR and PR. However, such an enhancement was offset by its low SUCRA values with respect to several adverse events.

\section{DISCUSSION}

The effectiveness of chemotherapy for managing melanoma patients is quite debatable due to the heterogeneity of tumors. Most melanoma patients are treated with either dacarbazine (DTIC) or temozolomide which contributes to a low objective response rate of less than $15 \%$ [38]. The development of targeted therapy aimed at gene mutations and aberrant cell signaling pathways appears to somewhat complement the standard chemotherapy. Melanoma patients treated with targeted therapy benefit from substantial increase in the overall response rate as well as extended survival [39-41]. Unfortunately, most melanoma patients develop resistance to targeted therapies and eventually experience metastatic tumor lesions [39, 40]. On the other hand, modest and long-lasting responses have been shown by antibodies which target CTLA-4 or PD-1. Unlike targeted therapy, more modest but durable responses were observed with patients treated with immune checkpoint inhibitors. However, such a durable response is not reflected by all patients due to significant heterogeneity in lymphocyte infiltration [38].

Immune checkpoint blockade antibodies targeting CTLA-4 and PD-1 have become a major breakthrough in cancer treatments for some patients with advanced melanoma. Compared to chemotherapy and molecularly targeted therapy, immune checkpoint therapy is able to provide more durable clinical response through the induction and activation of tumor-specific cytotoxic $\mathrm{T}$ cells [42]. It is well known that immune checkpoints have two important roles: maintenance of self-tolerance as 
Table 3: Main adverse event NMA outcomes for melanoma

\begin{tabular}{|c|c|c|c|c|c|c|c|c|}
\hline \multirow[t]{8}{*}{ Fatigue } & $\mathbf{A}$ & $1.29(0.48,3.34)$ & $0.85(0.24,3.22)$ & $1.32(0.56,2.90)$ & $0.62(0.15,1.88)$ & $0.64(0.20,2.74)$ & $1.47(0.41,4.66)$ & $\begin{array}{c}3.07(0.68, \\
13.80)\end{array}$ \\
\hline & $0.78(0.30,2.09)$ & B & $0.66(0.13,3.29)$ & $1.03(0.38,2.68)$ & $0.49(0.08,1.98)$ & $0.50(0.12,2.87)$ & $1.13(0.43,2.81)$ & $2.37(0.77,7.35)$ \\
\hline & $1.17(0.31,4.18)$ & $1.51(0.30,7.49)$ & $\mathrm{C}$ & $1.55(0.32,6.79)$ & $0.73(0.10,3.64)$ & $0.75(0.14,5.42)$ & $1.71(0.27,9.63)$ & $3.58(0.49,23.7)$ \\
\hline & $0.76(0.34,1.77)$ & $0.97(0.37,2.63)$ & $0.65(0.15,3.17)$ & D & $0.47(0.09,1.87)$ & $0.48(0.12,2.62)$ & $1.11(0.36,3.37)$ & $2.32(0.52,10.7)$ \\
\hline & $1.60(0.53,6.71)$ & $2.05(0.51,11.89)$ & $1.37(0.27,9.95)$ & $2.11(0.53,10.9)$ & $\mathbf{E}$ & $1.05(0.43,4.14)$ & $2.36(0.45,14.9)$ & $4.95(0.77,39.6)$ \\
\hline & $1.56(0.36,4.90)$ & $2.01(0.35,8.49)$ & $1.34(0.18,7.16)$ & $2.07(0.38,8.12)$ & $0.95(0.24,2.35)$ & $\mathbf{F}$ & $2.31(0.32,10.9)$ & $4.75(0.58,29.7)$ \\
\hline & $0.68(0.21,2.45)$ & $0.88(0.36,2.34)$ & $0.58(0.10,3.65)$ & $0.90(0.30,2.82)$ & $0.42(0.07,2.20)$ & $0.43(0.09,3.11)$ & G & $2.12(0.50,9.48)$ \\
\hline & $0.33(0.07,1.47)$ & $0.42(0.14,1.31)$ & $0.28(0.04,2.06)$ & $0.43(0.09,1.91)$ & $0.20(0.03,1.29)$ & $0.21(0.03,1.71)$ & $0.47(0.11,2.01)$ & $\mathbf{H}$ \\
\hline \multirow[t]{8}{*}{ Pruritus } & $\mathbf{A}$ & $7.62(3.33,20.42)$ & $8.84(1.96,42.1)$ & $4.62(1.98,13.7)$ & $5.21(1.78,19.51)$ & $5.40(1.35,25.58)$ & $9.31(3.00,36.8)$ & $4.45(1.53,13.4)$ \\
\hline & $0.13(0.05,0.30)$ & B & $1.16(0.18,6.14)$ & $0.60(0.22,1.75)$ & $0.68(0.23,2.28)$ & $0.71(0.15,3.59)$ & $1.23(0.45,3.56)$ & $0.58(0.20,1.60)$ \\
\hline & $0.11(0.02,0.51)$ & $0.86(0.16,5.53)$ & $\mathbf{C}$ & $0.52(0.09,3.65)$ & $0.59(0.10,4.69)$ & $0.61(0.08,5.74)$ & $1.05(0.17,8.48)$ & $0.50(0.08,3.32)$ \\
\hline & $0.22(0.07,0.51)$ & $1.67(0.57,4.53)$ & $1.93(0.27,10.6)$ & D & $1.14(0.28,4.73)$ & $1.18(0.19,6.64)$ & $2.03(0.61,6.83)$ & $0.96(0.23,3.42)$ \\
\hline & $0.19(0.05,0.56)$ & $1.47(0.44,4.34)$ & $1.71(0.21,10.4)$ & $0.88(0.21,3.57)$ & $\mathbf{E}$ & $1.03(0.24,4.28)$ & $1.81(0.39,8.06)$ & $0.85(0.17,3.41)$ \\
\hline & $0.19(0.04,0.74)$ & $1.42(0.28,6.61)$ & $1.65(0.17,12.9)$ & $0.85(0.15,5.15)$ & $0.97(0.23,4.17)$ & $\mathbf{F}$ & $1.75(0.27,11.2)$ & $0.83(0.13,4.47)$ \\
\hline & $0.11(0.03,0.33)$ & $0.81(0.28,2.20)$ & $0.96(0.12,5.78)$ & $0.49(0.15,1.64)$ & $0.55(0.12,2.57)$ & $0.57(0.09,3.68)$ & G & $0.47(0.11,1.82)$ \\
\hline & $0.22(0.07,0.65)$ & $1.71(0.63,5.09)$ & $2.02(0.30,13.3)$ & $1.04(0.29,4.31)$ & $1.17(0.29,5.76)$ & $1.21(0.22,7.84)$ & $2.11(0.55,9.33)$ & $\mathbf{H}$ \\
\hline \multirow[t]{8}{*}{ Diarrhea } & $\mathbf{A}$ & $2.32(1.67,3.06)$ & $5.16(3.03,8.37)$ & $1.07(0.73,1.49)$ & $1.34(0.81,2.15)$ & $1.01(0.45,2.05)$ & $3.75(2.34,5.68)$ & $2.55(1.63,3.75)$ \\
\hline & $0.43(0.33,0.60)$ & B & $2.21(1.25,3.91)$ & $0.46(0.32,0.66)$ & $0.58(0.36,0.93)$ & $0.43(0.20,0.95)$ & $1.62(1.11,2.34)$ & $1.10(0.71,1.71)$ \\
\hline & $0.19(0.12,0.33)$ & $0.45(0.26,0.80)$ & $\mathrm{C}$ & $0.21(0.11,0.38)$ & $0.26(0.13,0.54)$ & $0.20(0.08,0.46)$ & $0.73(0.38,1.41)$ & $0.50(0.26,0.94)$ \\
\hline & $0.93(0.67,1.37)$ & $2.17(1.51,3.09)$ & $4.78(2.60,8.83)$ & D & $1.27(0.73,2.21)$ & $0.93(0.41,2.13)$ & $3.50(2.30,5.32)$ & $2.40(1.42,3.96)$ \\
\hline & $0.74(0.46,1.23)$ & $1.73(1.07,2.76)$ & $3.81(1.86,7.59)$ & $0.78(0.45,1.38)$ & $\mathbf{E}$ & $0.72(0.34,1.47)$ & $2.79(1.53,4.88)$ & $1.92(1.01,3.39)$ \\
\hline & $0.99(0.49,2.24)$ & $2.31(1.06,5.06)$ & $5.09(2.16,12.6)$ & $1.08(0.47,2.43)$ & $1.39(0.68,2.90)$ & $\mathbf{F}$ & $3.76(1.65,8.57)$ & $2.57(1.12,5.72)$ \\
\hline & $0.27(0.18,0.43)$ & $0.62(0.43,0.90)$ & $1.37(0.71,2.62)$ & $0.29(0.19,0.43)$ & $0.36(0.20,0.65)$ & $0.27(0.12,0.61)$ & G & $0.68(0.40,1.17)$ \\
\hline & $0.39(0.27,0.61)$ & $0.91(0.59,1.40)$ & $2.01(1.06,3.85)$ & $0.42(0.25,0.70)$ & $0.52(0.29,0.99)$ & $0.39(0.17,0.89)$ & $1.47(0.85,2.52)$ & $\mathbf{H}$ \\
\hline \multirow[t]{8}{*}{ Rush } & $\mathbf{A}$ & $3.79(2.26,7.22)$ & $0.84(0.35,2.06)$ & $3.40(1.83,8.80)$ & $3.03(1.43,7.22)$ & $3.06(1.10,8.36)$ & $6.45(3.20,18.2)$ & $3.78(1.85,8.05)$ \\
\hline & $0.26(0.14,0.44)$ & B & $0.22(0.07,0.61)$ & $0.90(0.49,2.03)$ & $0.79(0.37,1.72)$ & $0.80(0.26,2.15)$ & $1.70(0.94,3.74)$ & $0.99(0.49,1.95)$ \\
\hline & $1.19(0.49,2.88)$ & $4.45(1.63,13.9)$ & C & $4.03(1.47,16.6)$ & $3.58(1.15,13.1)$ & $3.62(0.93,13.7)$ & $7.57(2.67,31.8)$ & $4.51(1.44,14.4)$ \\
\hline & $0.29(0.11,0.55)$ & $1.11(0.49,2.03)$ & $0.25(0.06,0.68)$ & D & $0.87(0.30,2.18)$ & $0.89(0.22,2.67)$ & $1.91(0.88,4.09)$ & $1.11(0.39,2.53)$ \\
\hline & $0.33(0.14,0.70)$ & $1.27(0.58,2.70)$ & $0.28(0.08,0.87)$ & $1.15(0.46,3.34)$ & $\mathbf{E}$ & $1.04(0.37,2.31)$ & $2.19(0.86,6.44)$ & $1.28(0.46,3.31)$ \\
\hline & $0.33(0.12,0.91)$ & $1.24(0.47,3.82)$ & $0.28(0.07,1.07)$ & $1.13(0.37,4.52)$ & $0.97(0.43,2.67)$ & $\mathbf{F}$ & $2.14(0.71,8.59)$ & $1.25(0.39,4.19)$ \\
\hline & $0.16(0.05,0.31)$ & $0.59(0.27,1.06)$ & $0.13(0.03,0.37)$ & $0.52(0.24,1.13)$ & $0.46(0.16,1.16)$ & $0.47(0.12,1.40)$ & G & $0.59(0.20,1.35)$ \\
\hline & $0.26(0.12,0.54)$ & $1.01(0.51,2.05)$ & $0.22(0.07,0.69)$ & $0.90(0.40,2.57)$ & $0.78(0.30,2.18)$ & $0.80(0.24,2.55)$ & $1.70(0.74,5.05)$ & $\mathbf{H}$ \\
\hline \multirow[t]{8}{*}{ Nausea } & $\mathbf{A}$ & $0.89(0.19,4.10)$ & $0.53(0.07,3.55)$ & $0.36(0.10,1.26)$ & $0.22(0.03,1.76)$ & $0.11(0.01,0.86)$ & $1.06(0.16,6.82)$ & $1.67(0.16,13.2)$ \\
\hline & $1.12(0.24,5.32)$ & B & $0.60(0.05,7.22)$ & $0.41(0.09,1.82)$ & $0.25(0.02,3.31)$ & $0.12(0.01,1.68)$ & $1.21(0.29,5.02)$ & $1.89(0.35,8.61)$ \\
\hline & $1.88(0.28,14.0)$ & $1.67(0.14,19.5)$ & $\mathbf{C}$ & $0.68(0.07,7.21)$ & $0.41(0.03,7.74)$ & $0.20(0.01,3.75)$ & $2.00(0.14,31.1)$ & $3.18(0.15,57.9)$ \\
\hline & $2.75(0.79,10.2)$ & $2.44(0.55,10.8)$ & $1.46(0.14,14.31)$ & D & $0.60(0.05,6.80)$ & $0.30(0.02,3.56)$ & $2.92(0.55,16.6)$ & $4.59(0.49,34.8)$ \\
\hline & $4.61(0.57,36.5)$ & $4.05(0.30,53.2)$ & $2.45(0.13,40.0)$ & $1.66(0.15,20.1)$ & $\mathbf{E}$ & $0.50(0.06,4.37)$ & $4.91(0.30,88.74$ & $7.72(0.34,142)$ \\
\hline & $9.37(1.16,81.1)$ & $8.33(0.59,112)$ & $4.97(0.27,87.7)$ & $3.36(0.28,40.7)$ & $2.02(0.23,17.2)$ & $\mathbf{F}$ & $10.1(0.57,170)$ & $\begin{array}{c}15.47(0.68 \\
317)\end{array}$ \\
\hline & $0.94(0.15,6.18)$ & $0.83(0.20,3.44)$ & $0.50(0.03,7.14)$ & $0.34(0.06,1.83)$ & $0.20(0.01,3.29)$ & $0.10(0.01,1.75)$ & G & $1.56(0.17,11.9)$ \\
\hline & $0.60(0.08,6.24)$ & $0.53(0.12,2.87)$ & $0.31(0.02,6.65)$ & $0.22(0.03,2.05)$ & $0.13(0.01,2.90)$ & $0.06(0.00,1.47)$ & $0.64(0.08,5.91)$ & $\mathbf{H}$ \\
\hline
\end{tabular}

Intervention: A: Chemotherapy; B: Ipilimumab; C: Tremelimumab; D: Nivolumab; E: Pembrolizumab 10 mg/kg; F: Pembrolizumab 2 mg/kg; G:

Ipilimumab+Nivolumab; H: Ipilimumab+Chemotherapy. 
well as regulating the amplitude and duration of T cells. Theoretically, immune checkpoint blockade antibodies that block CTLA-4 and PD-1 are able to restore and enhance cytotoxic $\mathrm{T}$ cell responses against tumors which exhibit resistance to chemotherapy [42].

Ipilimumab is a human monoclonal antibody that blocks CTLA-4 and was considered as the first option for advanced metastatic melanoma based on phase II and III trials [22, 43]. Our study not only verified that Ipilimumab exhibits enhanced efficacy compared with chemotherapy but also concluded that the combined intervention of Ipilimumab + Nivolumab is more effective than Ipilimumab monotherapy. Our results are supported by two randomized trials which suggest that the combined intervention of Ipilimumab + Nivolumab is recommended for patients with unresectable metastatic melanoma

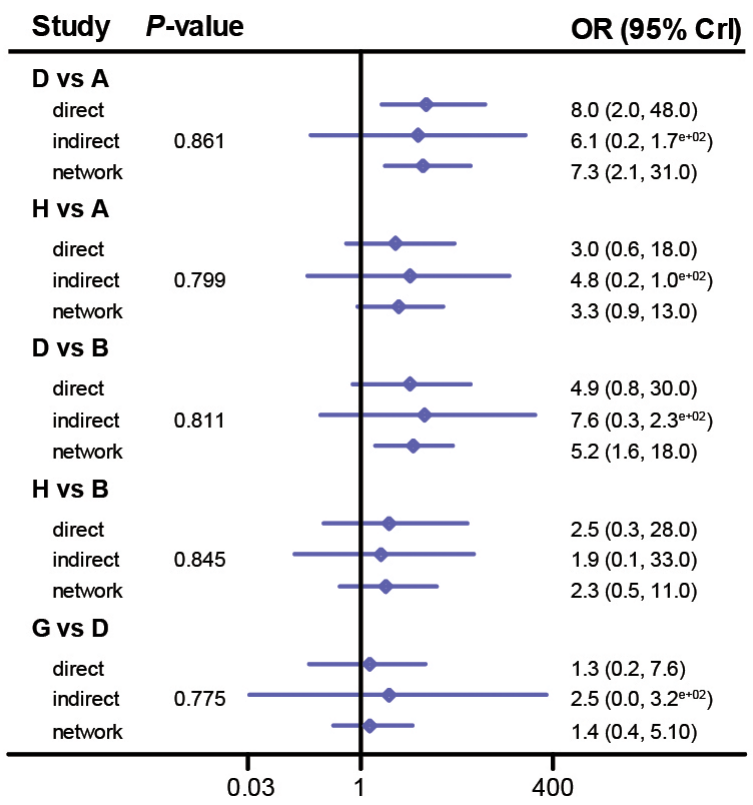

Figure 4: Node splitting plot of complete rate.

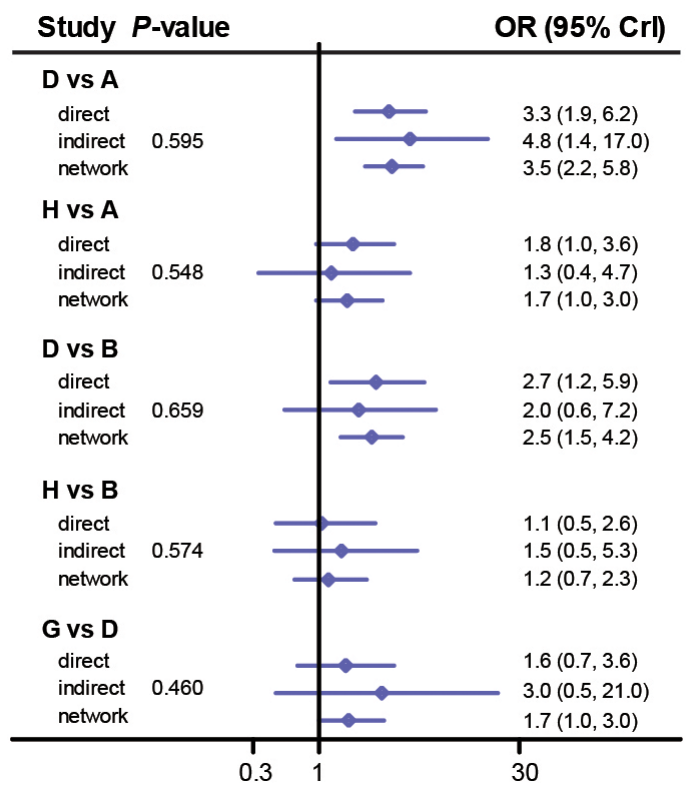

Figure 5: Node splitting plot of partial rate. 


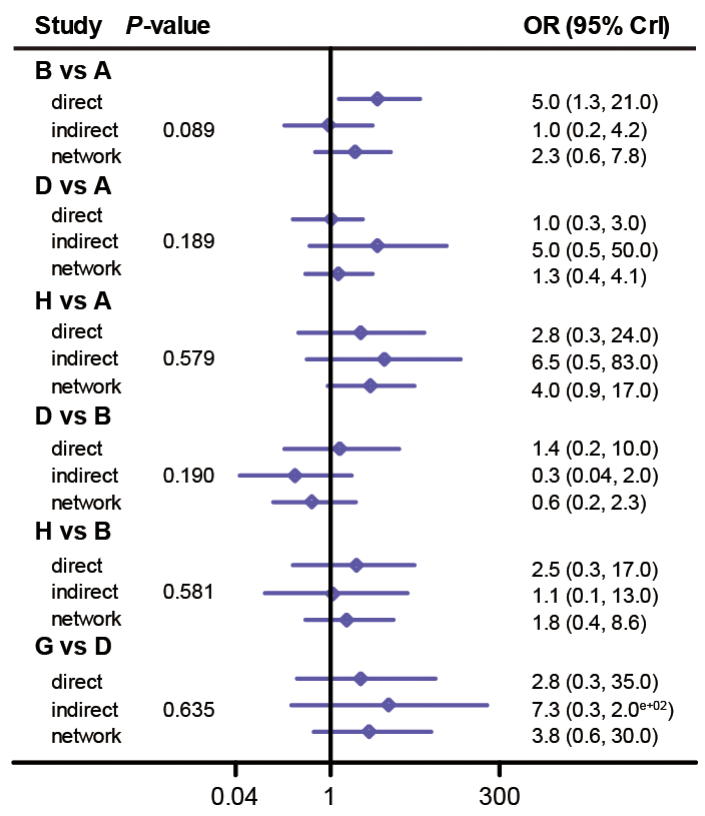

Figure 6: Node splitting plot of all adverse events.

Table 4: SUCRA results for eight intervention outcomes in treatments of melanoma

\begin{tabular}{lcccccccccc}
\hline Intervention & PFS & OS & CR & PR & AAE & Fatigue & Pruritus & Diarrhea & Rush & Nausea \\
\hline Chemotherapy & 0.042 & 0.053 & 0.097 & 0.089 & 0.643 & 0.583 & 0.996 & 0.856 & 0.896 & 0.286 \\
Ipilimumab & 0.414 & 0.839 & 0.219 & 0.306 & 0.143 & 0.411 & 0.296 & 0.380 & 0.337 & 0.363 \\
Tremelimumab & 0.718 & 0.258 & 0.220 & 0.129 & 0.393 & 0.646 & 0.266 & 0.021 & 0.941 & 0.529 \\
Nivolumab & 0.820 & 0.941 & 0.746 & 0.610 & 0.500 & 0.373 & 0.601 & 0.796 & 0.420 & 0.686 \\
Pembrolizumab 10 mg/kg & 0.321 & 0.380 & 0.729 & 0.873 & 0.964 & 0.820 & 0.526 & 0.641 & 0.513 & 0.770 \\
Pembrolizumab 2mg/kg & 0.456 & 0.494 & 0.669 & 0.786 & 0.893 & 0.771 & 0.494 & 0.837 & 0.486 & 0.916 \\
Ipilimumab+Nivolumab & 0.929 & & 0.857 & 0.866 & 0.000 & 0.326 & 0.197 & 0.136 & 0.037 & 0.280 \\
Ipilimumab+Chemotherapy & 0.301 & 0.534 & 0.479 & 0.341 & 0.464 & 0.084 & 0.614 & 0.320 & 0.359 & 0.169 \\
\hline
\end{tabular}

Outcomes: PFS: Progression Free Survival; OS: Overall Survival; CR: Complete Rate; PR: Partial Rate; AAE: All Adverse Events.

since they appear to have complementary activity in this disease [31]. Another phase II study in which patients with BRAF wild-type melanoma are included indicated that the combined strategy of Ipilimumab + Nivolumab is far more effective than Ipilimumab monotherapy since the corresponding $\mathrm{CR}$ rate for two treatment group were $22 \%$ and $0 \%$, respectively [33]. Therefore, introducing Nivolumab into Ipilimumab may enhance the effectiveness of Ipilimumab monotherapy.

Tremelimumab is another fully humanized monoclonal antibody targeting CTLA-4. Despite that some promising results have been observed in Phase I or II trials, Tremelimumab in phase III studies does not exhibit significant results for melanoma patients [10]. This may be explained by the fact that Tremelimumab binds to FcyR with lower affinity, which results in less effective blocking of CTLA-4 to T-cells [10]. As suggested by the corresponding ranking probabilities, Tremelimumab appears to be more effective than chemotherapy in PFS, OS, CR and PR. However, such a strength may be offset by its increased toxicity since patients treated with Tremelimumab are more likely to experience adverse events such as pruritus and diarrhea compared to those treated with chemotherapy. Monoclonal antibodies targeting CTLA-4 result in toxicities related to organspecific inflammatory processes [44]. Therefore, higher doses may result in both higher likelihood antitumor responses and toxicity. Nevertheless, our study does not 
(1) $\mathrm{CR}$

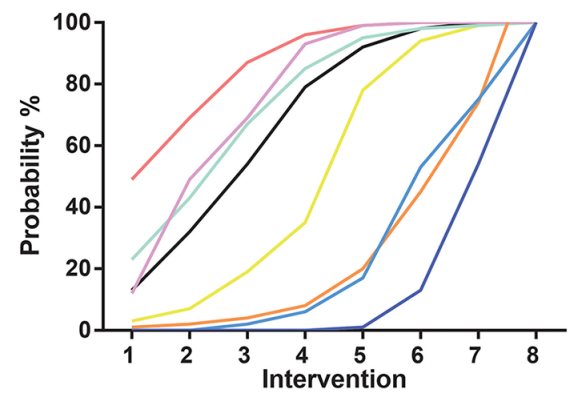

(3) AAE

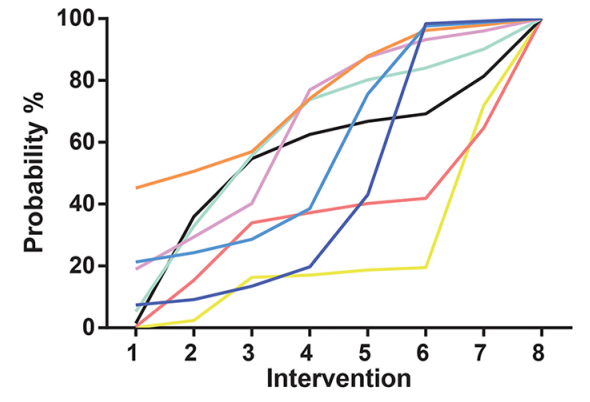

(5) Prurituse

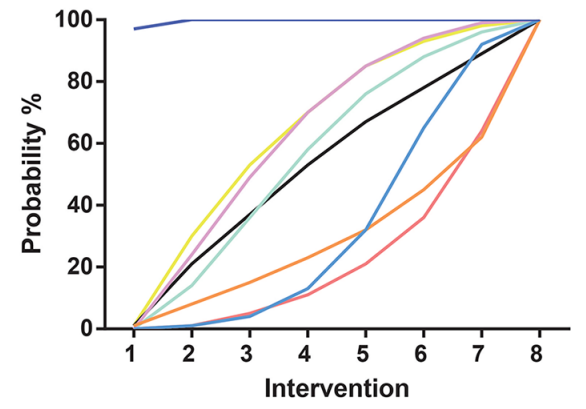

(7) Nausea

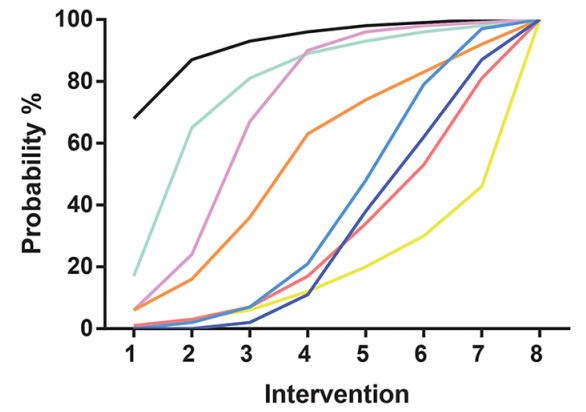

(2) PR

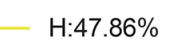

- G:87.71\%

- F: $66.86 \%$

- E: $72.86 \%$

- D:74.57\%

- C: $22.00 \%$

- B:21.86\%

- A: $9.71 \%$

- $\mathrm{H}: 20.82 \%$

- G:33.39\%

- F:53.14\%

- E:60.30\%

- D:63.18\%

- C: $72.72 \%$

- B:54.47\%

- A: $41.47 \%$

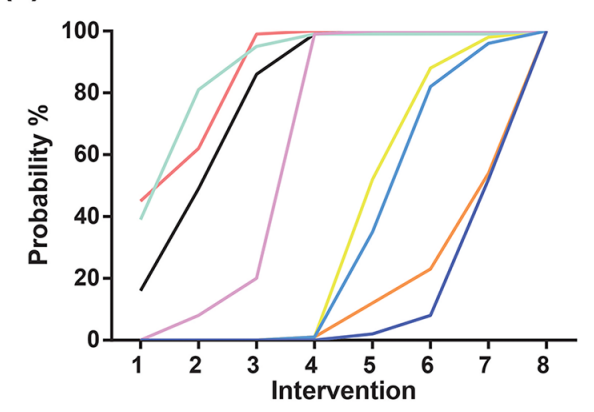

- $\mathrm{H}: 31.14 \%$

- G:86.57\%

- F: $78.57 \%$

- E:87.29\%

- D: $61.00 \%$

- C: $12.86 \%$

- B: $30.57 \%$

- A: $8.86 \%$

\section{(4) Fatigue}

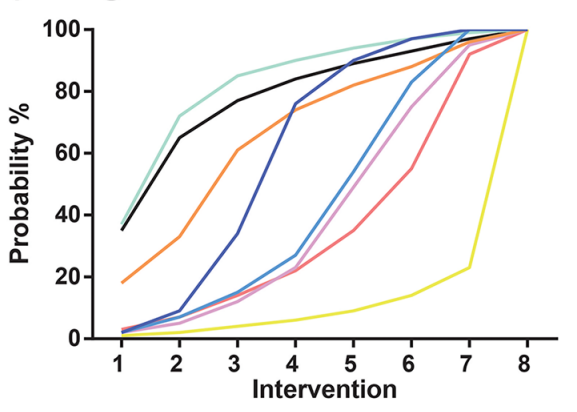

- $\mathrm{H}: 8.43 \%$

- G:32.57\%

- F: $77.14 \%$

- E:82.00\%

— D: $37.29 \%$

- C: $64.57 \%$

- B: $41.14 \%$

- A:58.29\%

\section{(6) Diarrhea}

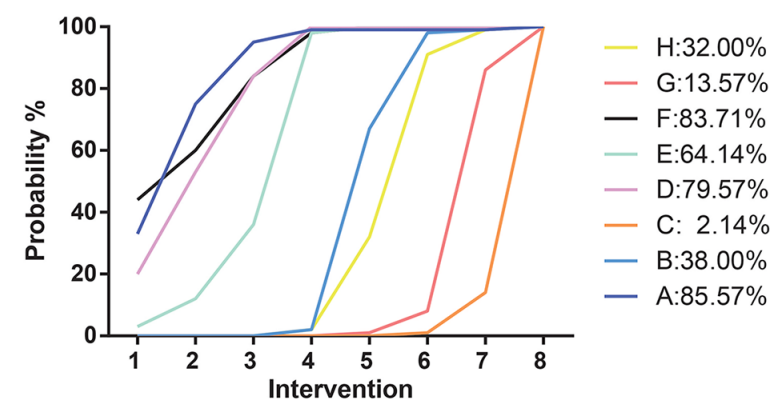

\section{(8) Rush}

- $\mathrm{H}: 16.86 \%$

- G:28.00\%

- F: $91.57 \%$

— E: $77.00 \%$

- D:68.57\%

- C:52.86\%

- B:36.29\%

- A:28.57\%

$\mathrm{H}: 35.86 \%$

- G: $3.71 \%$

- F: $48.57 \%$

- E:51.29\%

- D:42.00\%

- C: $94.14 \%$

- B:33.71\%

— A:89.57\%

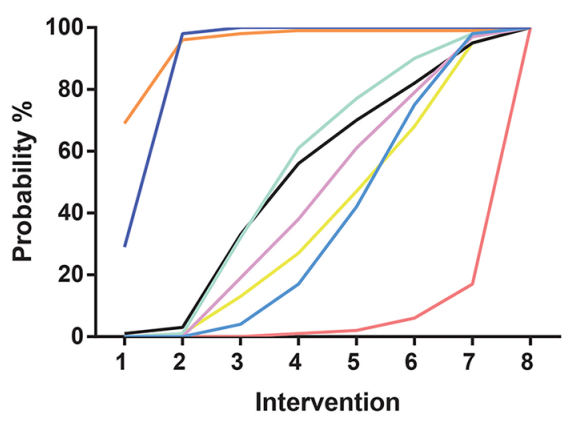

Figure 7: SUCRA of index except OS and PFS. (1) CR: complete rate; (2) PR: partial rate; (3) AAE: all adverse events; (4) Fatigue; (5) Pruritus; (6) Diarrhea; (7) Nausea; (8) Rush.

enable us to perform a stratified analysis by dose and such a mechanism should be thoroughly studied. However, it is still a challenging task for researchers to simultaneously assess how different dosages of melanoma interventions contribute to different levels of toxicity, because it may require biopsies of organs before and after applying interventions to patients, which is only feasible in the intestinal tract or the skin [44].

PD-1 is another checkpoint molecule that has a significant role in T-cell regulation and it suppresses 
the activity of T-cells in peripheral tissues when an inflammatory response is triggered [45]. Furthermore, PD-1 is a $55 \mathrm{kDa}$ type I transmembrane protein encoded by the $P D C D 1$ gene. This protein is comprised of several elements, including a extracellular immunoglobulin (Ig) domain, a transmembrane domain and an intracellular domain that contains phosphorylation sites [45]. Nivolumab is a fully human monoclonal IgG4 antibody that binds PD-1 with high affinity and it also prevents interaction with PD-L1 and PD-L2 which are two known ligands of PD-1. As suggested by in vitro assays, Nivolumab is able to increase both antigen-specific T-cell responses and cytokine production [46]. Moreover, Nivolumab is administered intravenously over a period of 60 minutes and its serum half-life approximately ranges from 12 days to 20days depending on the corresponding dosage [45]. Another feature of Nivolumab is its linear pharmacokinetics which is able to provide durable responses in patients with a wide range of advanced malignancies including melanoma, which has been verified in phase I trials [47]. Nivolumab is generally welltolerated by the majority of patients. The most common adverse events associated with Nivolumab include lowgrade fatigue, musculoskeletal side effects, decreased appetite, nausea, diarrhea, rash and pruritus [48]. In our study, patients treated with Nivolumab are associated with a significant increase in efficacy compared with those treated with chemotherapy. Nevertheless, this trend is accompanied with an increase in the likelihood of adverse events such as fatigue, pruritus, diarrhea and rush.

Our study not only compared different interventions for melanoma patients but also investigated how different dosage of Pembrolizumab affects its efficacy and tolerability. Currently, the recommended dose of Pembrolizumab for advanced melanoma patients is $2 \mathrm{mg} /$ $\mathrm{kg}$ and it is administered via intravenous infusion over 30 min every three weeks [15]. As suggested by a keynote trial in which a total of 655 patients were allocated to different treatment groups, Pembrolizumab is able to provide durable antitumor activity for patients who are refractory to Ipilimumab [29, 49]. Another randomized clinical trial which compared Pembrolizumab with chemotherapy indicated that melanoma patients treated with Pembrolizumab exhibit more desirable PFS status [50]. More importantly, a large phase III suggested that both PFS and OS status for advanced melanoma patients treated with Pembrolizumab were significantly prolonged compared to those treated with Ipilimumab [35, 50]. The above conclusion is consistent with the results obtained from our study which concluded that melanoma patients treated with Pembrolizumab have significantly higher CR and PR than those treated with chemotherapy or Ipilimumab. Moreover, autoimmune complications resulted from Pembrolizumab are fewer and less severe as compared to those treated with antibodies targeting CTLA-4 [50]. This conclusion is supported by our analysis since the corresponding
SUCRA values of Pembrolizumab $(10 \mathrm{mg} / \mathrm{kg}$ or $2 \mathrm{mg}$ / $\mathrm{kg}$ ) with respect to adverse events are significantly higher than those of the two CTLA-4 antibodies (Ipilimumab, Tremelimumab). Although there appears to be slight difference in the SUCRA values between different doses of Pembrolizumab, pooled statistics such as HR/OR between them do not differ significantly with respect to all endpoints. Generally, increasing the dosage of Pembrolizumab may not have significant effect on its effectiveness nor tolerability. A number of studies have been designed to discover the optimal dose of Pembrolizumab, but none of them showed significant results and therefore $2 \mathrm{mg} / \mathrm{kg}$ is still considered as the recommended dose for melanoma patients. In addition, it is also recommended that thyroid function should be routinely monitored in patients who are treated with Pembrolizumab.

However, only a portion of patients respond to immune checkpoint therapy. Identifying patients who are most likely to respond to each immune checkpoint therapy is critical to the selection of interventions. However, this task cannot be achieved by simply comparing the effectiveness and tolerability of different interventions since randomized trials are usually carried out in different populations. On top of that, we do not have any knowledge about whether prior therapies had been applied to these melanoma patients and such a limitation may consequently affect the effectiveness and tolerability of immune checkpoint therapies. Also, formalized stopping rules which determine the corresponding treatment duration for each immune checkpoint therapy are not provided in most of the studies and hence it is challenging to determine the optimal duration for each immune checkpoint therapy. It is likely that the selection of treatments may be influenced by treatment schedule and costs which may distort the effect of randomization in clinical trials.

Overall, our study provides solid evidence that several immune checkpoint therapies or combined interventions may be more effective than chemotherapy for managing melanoma patients. However, chemotherapy appears to be more tolerable than these combined strategies since patients are less likely to experience adverse events. In the future, we encourage researchers to conduct ongoing clinical trials in which confounding factors are controlled in order to prevent biased results.

\section{MATERIALS AND METHODS}

\section{Search strategy}

We began our research with the formulation of a comprehensive searching strategy. A thorough literature search was conducted in PubMed, Embase and Cochrane Library. Firstly, we searched previous systematic reviews and NMA in order to ensure that this topic has not been carried out in the current literature. The entire searching strategy was carried out by two independent researchers 
without limitations to identify studies about pharmacological interventions for melanoma. Search results were compared and the differences were resolved by discussion. Disagreements after discussion were viewed and validated by a third independent researcher. Additional articles were obtained by scanning reference lists of relevant studies. The entire research question was break down into small pieces so that the scope of the question can be clearly defined.

\section{Inclusion criteria and screen of individual studies}

A few guidelines were created for screening eligible studies: 1) study design must be randomized clinical trials with subjects older than 18 years; 2) at least one comparison was made among interventions including chemotherapy, Ipilimumab, Tremelimumab, Nivolumab, Pembrolizumab (10 mg/kg or $2 \mathrm{mg} / \mathrm{kg})$, Ipilimumab + Nivolumab and Ipilimumab + Chemotherapy; 3) At least one of the following endpoints were evaluated by the study, including progression free survival (PFS), overall survival (OS), complete response (CR), partial response (PR), all adverse events (AAE), fatigue, pruritus, diarrhea, rush and nausea; 4) the corresponding data required for NMA must be available within the study. We used PICOS approach in this study and the detailed PICOS criteria we followed are specified in Supplementary Table S2. Individual studies that does not comply with the selection criteria were excluded.

\section{Data extraction and quality assessment}

Data extraction and synthesis was conducted by two reviewers independently by using a data extraction spreadsheet. The following data were extracted and recorded from eligible studies: author, intervention, dosage, study sample size, average age of subjects, percentage of males, metastasis status and the corresponding endpoints. Moreover, interventions are carefully selected in order to form a closed loop for the implementation of NMA. Endpoints were clearly defined in the following ways so that selection bias can be minimized: PFS (time between the start of treatment and documented disease progression or death due to any cause); OS (time between the start of treatment and death due to any cause); CR (disappearance of all target lesions in response to treatment); PR (more than 30\% decrease in the sum of diameters of target lesions in response to treatment).

The quality of included randomized controlled trials was evaluated by the Jadad scale [51]. Randomization, blinding and withdrawal were used as three scoring items.

\section{Statistical analysis}

We carried out the NMA by using the Bayesian conceptual framework which incorporates a prior probability distribution, a likelihood function based on data and a posterior probability distribution that combines the first two elements [52]. Comparisons between interventions with respect to OS and PFS were achieved by computing the statistics of hazard ratio (HR) with $95 \%$ confidential intervals (CrIs). The odds ratio (OR) with 95\% CrIs was used to compare interventions with respect to other endpoints. Furthermore, the nodesplitting method is adopted to evaluate the extent of consistency between direct and indirect evidence within the network [37]. The ranking of interventions with respect to each endpoint is performed by using the corresponding surface under the cumulative ranking area (SUCRA) introduced by Salanti et al [53]. The SCURA is a useful numerical summary and allows to identify the probability of being best, being worst etc. The larger the SUCRA value, the better the rank of the treatment. Between-study heterogeneity which may arise from different sources of study variability is assessed by the Cochran's $Q$ test and the degree of heterogeneity is quantified by the statistics of $I^{2}$. Finally, publication bias is visually assessed by using the funnel plot in which asymmetry pattern may provide evidence for publication bias. All statistical procedures were implemented using WinBugs (MRC Biostatistics Unit) and R software (Version 3.2.4) with package 'gemtc' (version 0.8).

\section{Abbreviations}

network meta-analysis, NMA; overall survival, OS; progression free survival, PFS; complete response, CR; partial response, $\mathrm{PR}$; hazard ratio, $\mathrm{HR}$; odds ratio, OR; cytotoxic T-lymphocyte antigen-4, CTLA-4; programmed death 1, PD1; surface under the cumulative ranking area, SUCRA

\section{ACKNOWLEDGMENTS}

It's funded by the National Natural Science Foundation of Major Projects Overseas of China (Grant No. 81120108008) and the National Natural Science Foundation of China (Grant No. 81071581).

\section{CONFLICTS OF INTEREST}

The authors declare no conflict of interest.

\section{REFERENCES}

1. Ferlay J, Steliarova-Foucher E, Lortet-Tieulent J, Rosso S, Coebergh JW, Comber H, Forman D and Bray F. Cancer incidence and mortality patterns in Europe: estimates for 40 countries in 2012. Eur J Cancer. 2013; 49:1374-1403.

2. Bender C, Hassel JC and Enk A. Immunotherapy of Melanoma. Oncology research and treatment. 2016; 39:369-376. 
3. Nevala WK, Buhrow SA, Knauer DJ, Reid JM, Atanasova EA and Markovic SN. Antibody Targeted Chemotherapy for the Treatment of Melanoma. Cancer research. 2016.

4. van Akkooi AC, Atkins MB, Agarwala SS and Lorigan P. Surgical Management and Adjuvant Therapy for High-Risk and Metastatic Melanoma. American Society of Clinical Oncology educational book / ASCO American Society of Clinical Oncology Meeting. 2016; 35:e505-514.

5. Gasser M and Waaga-Gasser AM. Therapeutic Antibodies in Cancer Therapy. Advances in experimental medicine and biology. 2016; 917:95-120.

6. Hodi FS, O'Day SJ, McDermott DF, Weber RW, Sosman JA, Haanen JB, Gonzalez R, Robert C, Schadendorf D, Hassel JC, Akerley W, van den Eertwegh AJ, Lutzky $\mathrm{J}$, et al. Improved survival with ipilimumab in patients with metastatic melanoma. The New England journal of medicine. 2010; 363:711-723.

7. Wolchok JD, Weber JS, Maio M, Neyns B, Harmankaya K, Chin K, Cykowski L, de Pril V, Humphrey R and Lebbe C. Four-year survival rates for patients with metastatic melanoma who received ipilimumab in phase II clinical trials. Annals of oncology. 2013; 24:2174-2180.

8. Joseph RW, Cappel M, Tzou K, Bagaria S, Gilstrap C, Swaika A and Jambusaria-Pahlajani A. Treatment of in-transit and metastatic melanoma in two patients treated with ipilimumab and topical imiquimod. Melanoma research. 2016.

9. Postow MA, Callahan MK, Barker CA, Yamada Y, Yuan J, Kitano S, Mu Z, Rasalan T, Adamow M, Ritter E, Sedrak C, Jungbluth AA, Chua R, et al. Immunologic correlates of the abscopal effect in a patient with melanoma. The New England journal of medicine. 2012; 366:925-931.

10. Comin-Anduix B, Escuin-Ordinas $\mathrm{H}$ and Ibarrondo FJ. Tremelimumab: research and clinical development. OncoTargets and therapy. 2016; 9:1767-1776.

11. Wang E, Kang D, Bae KS, Marshall MA, Pavlov $\mathrm{D}$ and Parivar K. Population pharmacokinetic and pharmacodynamic analysis of tremelimumab in patients with metastatic melanoma. Journal of clinical pharmacology. 2014; 54:1108-1116.

12. Watanabe S, Kimura H, Takato H, Waseda Y, Hara J, Sone T, Abo M, Maeda S, Matsushita T and Kasahara K. Severe pneumonitis after nivolumab treatment in a patient with melanoma. Allergology international. 2016.

13. Orloff M, Weight $\mathrm{R}$, Valsecchi ME and Sato T. Immune Check Point Inhibitors Combination in Melanoma: Worth the Toxicity? Reviews on recent clinical trials. 2016; 11:81-86.

14. Kourie HR and Klastersky JA. Side-effects of checkpoint inhibitor-based combination therapy. Current opinion in oncology. 2016; 28:306-313.
15. Daud A and Nandoskar P. Pembrolizumab for melanomasafety profile and future trends. Expert opinion on drug safety. 2016; 15:727-729.

16. Pardoll DM. Immunology beats cancer: a blueprint for successful translation. Nature immunology. 2012; 13:1129-1132.

17. Ribas A, Puzanov I, Dummer R, Schadendorf D, Hamid O, Robert C, Hodi FS, Schachter J, Pavlick AC, Lewis KD, Cranmer LD, Blank CU, O'Day SJ, et al. Pembrolizumab versus investigator-choice chemotherapy for ipilimumabrefractory melanoma (KEYNOTE-002): a randomised, controlled, phase 2 trial. The Lancet Oncology. 2015; 16:908-918.

18. Ribas A, Hamid O, Daud A, Hodi FS, Wolchok JD, Kefford R, Joshua AM, Patnaik A, Hwu WJ, Weber JS, Gangadhar TC, Hersey P, Dronca R, et al. Association of Pembrolizumab With Tumor Response and Survival Among Patients With Advanced Melanoma. Jama. 2016; 315:1600-1609.

19. Ribas A, Camacho LH, Lopez-Berestein G, Pavlov D, Bulanhagui CA, Millham R, Comin-Anduix B, Reuben JM, Seja E, Parker CA, Sharma A, Glaspy JA and Gomez-Navarro J. Antitumor activity in melanoma and anti-self responses in a phase I trial with the anticytotoxic T lymphocyte-associated antigen 4 monoclonal antibody CP-675,206. Journal of clinical oncology. 2005; 23:8968-8977.

20. Camacho LH, Antonia S, Sosman J, Kirkwood JM, Gajewski TF, Redman B, Pavlov D, Bulanhagui C, Bozon VA, GomezNavarro J and Ribas A. Phase I/II trial of tremelimumab in patients with metastatic melanoma. Journal of clinical oncology. 2009; 27:1075-1081.

21. Weber J, Thompson JA, Hamid O, Minor D, Amin A, Ron I, Ridolfi R, Assi H, Maraveyas A, Berman D, Siegel J and O'Day SJ. A randomized, double-blind, placebo-controlled, phase II study comparing the tolerability and efficacy of ipilimumab administered with or without prophylactic budesonide in patients with unresec Supplementary Table Stage III or IV melanoma. Clinical cancer research. 2009; 15:5591-5598.

22. Wolchok JD, Neyns B, Linette G, Negrier S, Lutzky J, Thomas L, Waterfield W, Schadendorf D, Smylie M, Guthrie T, Jr., Grob JJ, Chesney J, Chin K, et al. Ipilimumab monotherapy in patients with pretreated advanced melanoma: a randomised, double-blind, multicentre, phase 2, dose-ranging study. The Lancet Oncology. 2010; 11:155-164.

23. Hamid O, Schmidt H, Nissan A, Ridolfi L, Aamdal S, Hansson J, Guida M, Hyams DM, Gomez H, Bastholt L, Chasalow SD and Berman D. A prospective phase II trial exploring the association between tumor microenvironment biomarkers and clinical activity of ipilimumab in advanced melanoma. Journal of translational medicine. 2011; 9:204. 
24. Hersh EM, O'Day SJ, Powderly J, Khan KD, Pavlick AC, Cranmer LD, Samlowski WE, Nichol GM, Yellin MJ and Weber JS. A phase II multicenter study of ipilimumab with or without dacarbazine in chemotherapy-naïve patients with advanced melanoma. Investigational New Drugs. 2011; 29:489-498.

25. Robert C, Thomas L, Bondarenko I, O'Day S, Weber J, Garbe C, Lebbe C, Baurain JF, Testori A, Grob JJ, Davidson $\mathrm{N}$, Richards J, Maio M, et al. Ipilimumab plus dacarbazine for previously untreated metastatic melanoma. The New England journal of medicine. 2011; 364:2517-2526.

26. Millward M, Underhill C, Lobb S, McBurnie J, Meech SJ, Gomez-Navarro J, Marshall MA, Huang B and Mather CB. Phase I study of tremelimumab (CP-675 206) plus PF-3512676 (CPG 7909) in patients with melanoma or advanced solid tumours. British journal of cancer. 2013; 108:1998-2004.

27. Ribas A, Kefford R, Marshall MA, Punt CJ, Haanen JB, Marmol M, Garbe C, Gogas H, Schachter J, Linette G, Lorigan P, Kendra KL, Maio M, et al. Phase III randomized clinical trial comparing tremelimumab with standard-ofcare chemotherapy in patients with advanced melanoma. Journal of clinical oncology. 2013; 31:616-622.

28. Hodi FS, Lee S, McDermott DF, Rao UN, Butterfield LH, Tarhini AA, Leming P, Puzanov I, Shin D and Kirkwood JM. Ipilimumab plus sargramostim vs ipilimumab alone for treatment of metastatic melanoma: a randomized clinical trial. Jama. 2014; 312:1744-1753.

29. Robert C, Ribas A, Wolchok JD, Hodi FS, Hamid O, Kefford R, Weber JS, Joshua AM, Hwu WJ, Gangadhar TC, Patnaik A, Dronca R, Zarour H, et al. Anti-programmeddeath-receptor-1 treatment with pembrolizumab in ipilimumab-refractory advanced melanoma: a randomised dose-comparison cohort of a phase 1 trial. Lancet (London, England). 2014; 384:1109-1117.

30. Eggermont AM, Chiarion-Sileni V, Grob JJ, Dummer R, Wolchok JD, Schmidt H, Hamid O, Robert C, Ascierto PA, Richards JM, Lebbe C, Ferraresi V, Smylie M, et al. Adjuvant ipilimumab versus placebo after complete resection of high-risk stage III melanoma (EORTC 18071): a randomised, double-blind, phase 3 trial. The Lancet Oncology. 2015; 16:522-530.

31. Larkin J, Chiarion-Sileni V, Gonzalez R, Grob JJ, Cowey CL, Lao CD, Schadendorf D, Dummer R, Smylie M, Rutkowski P, Ferrucci PF, Hill A, Wagstaff J, et al. Combined Nivolumab and Ipilimumab or Monotherapy in Untreated Melanoma. The New England journal of medicine. 2015; 373:23-34.

32. Maio M, Grob JJ, Aamdal S, Bondarenko I, Robert C, Thomas L, Garbe C, Chiarion-Sileni V, Testori A, Chen TT, Tschaika M and Wolchok JD. Five-year survival rates for treatment-naive patients with advanced melanoma who received ipilimumab plus dacarbazine in a phase III trial. Journal of clinical oncology. 2015; 33:1191-1196.
33. Postow MA, Chesney J, Pavlick AC, Robert C, Grossmann K, McDermott D, Linette GP, Meyer N, Giguere JK, Agarwala SS, Shaheen M, Ernstoff MS, Minor D, et al. Nivolumab and ipilimumab versus ipilimumab in untreated melanoma. The New England journal of medicine. 2015; 372:2006-2017.

34. Robert C, Long GV, Brady B, Dutriaux C, Maio M, Mortier L, Hassel JC, Rutkowski P, McNeil C, KalinkaWarzocha E, Savage KJ, Hernberg MM, Lebbe C, et al. Nivolumab in previously untreated melanoma without BRAF mutation. The New England journal of medicine. 2015; 372:320-330.

35. Robert C, Schachter J, Long GV, Arance A, Grob JJ, Mortier L, Daud A, Carlino MS, McNeil C, Lotem M, Larkin J, Lorigan P, Neyns B, et al. Pembrolizumab versus Ipilimumab in Advanced Melanoma. The New England journal of medicine. 2015; 372:2521-2532.

36. Weber JS, D'Angelo SP, Minor D, Hodi FS, Gutzmer R, Neyns B, Hoeller C, Khushalani NI, Miller WH, Jr., Lao CD, Linette GP, Thomas L, Lorigan P, et al. Nivolumab versus chemotherapy in patients with advanced melanoma who progressed after anti-CTLA-4 treatment (CheckMate 037): a randomised, controlled, open-label, phase 3 trial. The Lancet Oncology. 2015; 16:375-384.

37. van Valkenhoef $G$, Dias $S$, Ades $A E$ and Welton NJ. Automated generation of node-splitting models for assessment of inconsistency in network meta-analysis. Research synthesis methods. 2016; 7:80-93.

38. Somasundaram R and Herlyn M. Nivolumab in combination with ipilimumab for the treatment of melanoma. Expert review of anticancer therapy. 2015; 15:1135-1141.

39. Lo JA and Fisher DE. The melanoma revolution: from UV carcinogenesis to a new era in therapeutics. Science. 2014; 346:945-949.

40. Shtivelman E, Davies MQ, Hwu P, Yang J, Lotem M, Oren M, Flaherty KT and Fisher DE. Pathways and therapeutic targets in melanoma. Oncotarget. 2014; 5:1701-1752. doi: 10.18632/oncotarget.1892.

41. Schadendorf D, Fisher DE, Garbe C, Gershenwald JE, Grob JJ, Halpern A, Herlyn M, Marchetti MA, McArthur G, Ribas A, Roesch A and Hauschild A. Melanoma. Nature reviews Disease primers. 2015; 1:15003.

42. Ma W, Gilligan BM, Yuan J and Li T. Current status and perspectives in translational biomarker research for PD-1/ PD-L1 immune checkpoint blockade therapy. Journal of hematology \& oncology. 2016; 9:47.

43. Hersh EM, O’Day SJ, Powderly J, Khan KD, Pavlick AC, Cranmer LD, Samlowski WE, Nichol GM, Yellin MJ and Weber JS. A phase II multicenter study of ipilimumab with or without dacarbazine in chemotherapy-naive patients with advanced melanoma. Invest New Drugs. 2011; 29:489-498.

44. Ribas A. Anti-CTLA4 Antibody Clinical Trials in Melanoma. Update on cancer therapeutics. 2007; 2:133-139. 
45. Asmar R, Yang $\mathrm{J}$ and Carvajal RD. Clinical utility of nivolumab in the treatment of advanced melanoma. Therapeutics and clinical risk management. 2016; 12:313-325.

46. Wang C, Thudium KB, Han M, Wang XT, Huang H, Feingersh D, Garcia C, Wu Y, Kuhne M, Srinivasan $\mathrm{M}$, Singh S, Wong $\mathrm{S}$, Garner $\mathrm{N}$, et al. In vitro characterization of the anti-PD-1 antibody nivolumab, BMS-936558, and in vivo toxicology in non-human primates. Cancer immunology research. 2014; 2:846-856.

47. Topalian SL, Hodi FS, Brahmer JR, Gettinger SN, Smith DC, McDermott DF, Powderly JD, Carvajal RD, Sosman JA, Atkins MB, Leming PD, Spigel DR, Antonia SJ, et al. Safety, activity, and immune correlates of anti-PD-1 antibody in cancer. The New England journal of medicine. 2012; 366:2443-2454.

48. Brahmer JR, Drake CG, Wollner I, Powderly JD, Picus J, Sharfman WH, Stankevich E, Pons A, Salay TM, McMiller TL, Gilson MM, Wang C, Selby M, et al. Phase I study of single-agent anti-programmed death-1 (MDX1106) in refractory solid tumors: safety, clinical activity, pharmacodynamics, and immunologic correlates. Journal of clinical oncology. 2010; 28:3167-3175.

49. Patnaik A, Kang SP, Rasco D, Papadopoulos KP, ElassaissSchaap J, Beeram M, Drengler R, Chen C, Smith L, Espino G, Gergich K, Delgado L, Daud A, et al. Phase I Study of Pembrolizumab (MK-3475; Anti-PD-1 Monoclonal Antibody) in Patients with Advanced Solid Tumors. Clinical cancer research. 2015; 21:4286-4293.

50. Daud A. Current and Emerging Perspectives on Immunotherapy for Melanoma. Seminars in oncology. 2015; 42:S3-S11.

51. Jadad AR, Moore RA, Carroll D, Jenkinson C, Reynolds DJM, Gavaghan DJ and McQuay HJ. Assessing the quality of reports of randomized clinical trials: is blinding necessary? Controlled clinical trials. 1996; 17:1-12.

52. Lu G and Ades AE. Combination of direct and indirect evidence in mixed treatment comparisons. Statistics in medicine. 2004; 23:3105-3124.

53. Salanti G, Ades AE and Ioannidis JPA. Graphical methods and numerical summaries for presenting results from multiple-treatment meta-analysis: an overview and tutorial. Journal of Clinical Epidemiology. 64:163-171. 